\title{
Multiple Positive Solutions for Nonlocal Elliptic Problems Involving the Hardy Potential and Concave-Convex Nonlinearities
}

\author{
Shaya Shakerian \\ Department of Mathematics \\ University of British Columbia \\ Vancouver BC Canada V6T 1Z2 \\ shaya@math.ubc.ca
}

October 22, 2018

\begin{abstract}
In this paper, we study the existence and multiplicity of solutions for the following fractional problem involving the Hardy potential and concave-convex nonlinearities:

$$
\left\{\begin{aligned}
(-\Delta)^{\frac{\alpha}{2}} u-\gamma \frac{u}{|x|^{\alpha}} & =\lambda f(x)|u|^{q-2} u+g(x) \frac{|u|^{p-2} u}{|x|^{s}} & & \text { in } \Omega \\
u & =0 & & \text { in } \mathbb{R}^{n} \backslash \Omega,
\end{aligned}\right.
$$

where $\Omega \subset \mathbb{R}^{n}$ is a smooth bounded domain in $\mathbb{R}^{n}$ containing 0 in its interior, and $f, g \in C(\bar{\Omega})$ with $f^{+}, g^{+} \not \equiv 0$ which may change sign in $\bar{\Omega}$. We use the variational methods and the Nehari manifold decomposition to prove that this problem has at least two positive solutions for $\lambda$ sufficiently small. The variational approach requires that $0<\alpha<2,0<s<\alpha<n, 1<q<2<p \leq 2_{\alpha}^{*}(s):=\frac{2(n-s)}{n-\alpha}$, and $\gamma<\gamma_{H}(\alpha)$, the latter being the best fractional Hardy constant on $\mathbb{R}^{n}$.
\end{abstract}

\section{Introduction}

In this paper, we investigate the multiplicity results of positive solutions for the following fractional elliptic problem involving the Hardy potential and concave-convex non-linearities:

$$
\left\{\begin{aligned}
(-\Delta)^{\frac{\alpha}{2}} u-\gamma \frac{u}{|x|^{\alpha}} & =\lambda f(x)|u|^{q-2} u+g(x) \frac{|u|^{p-2} u}{|x|^{s}} & & \text { in } \Omega \\
u & =0 & & \text { in } \mathbb{R}^{n} \backslash \Omega,
\end{aligned}\right.
$$

where $\Omega \subset \mathbb{R}^{n}$ is a smooth bounded domain in $\mathbb{R}^{n}$ containing 0 in its interior, $0<\alpha<2,0<s<$ $\alpha<n, 1<q<2<p \leq 2_{\alpha}^{*}(s):=\frac{2(n-s)}{n-\alpha}, \gamma<\gamma_{H}(\alpha)=2^{\alpha} \frac{\Gamma^{2}\left(\frac{n+\alpha}{4}\right)}{\Gamma^{2}\left(\frac{n-\alpha}{4}\right)}$, the later being the best fractional Hardy constant on $\mathbb{R}^{n}$, and $f, g \in C(\bar{\Omega})$ with $f^{+}, g^{+} \not \equiv 0$ (they are possibly change sign in $\bar{\Omega}$ ). 
Addressing the questions regarding the effect of concave-convex non-linearities on the number of positive solutions for non-local elliptic problems has been the subject of several studies; see [1-4] and [17. Barrios-Medina-Peral [4] studied the sub-critical case of (1), and proved that there exists $\Lambda>0$ such that the problem has at least two solutions for all $0<\lambda<\Lambda$ when $f(x)=g(x) \equiv 1, s=0$, and $\gamma<\gamma_{H}(\alpha)$. The same results have been obtained by Barrios et al. in [2] in the critical case, but in the absence of the Hardy and singularity terms, i.e., when $\gamma=s=0$. Recently, Zhang-Liu-Jiao 25] extended the results of [2] to the problem involving the sign-changing weight function $f(x) \in C(\bar{\Omega})$ with $f^{+} \not \equiv 0$, and $g(x) \equiv 1$.

When $\alpha=2$, i.e., in the case of the standard Laplacian, problem (11) has been studied extensively in the last decade; see for example [18, [19, 20], 24] and references therein.

We point out that the non-local problems are still much less understood than their local counterpart. The aim of this paper is to consider the remaining cases and generalize the results of [4] and 25] to the problem involving the Hardy potential, Hardy-Sobolev singularity term, and also signchanging functions $f(x)$ and $g(x)$. More pricesly, we study problem (11) in the sub-critical (i.e., when $2<p<2_{\alpha}^{*}(s)$ ) and critical (i.e., when $p=2_{\alpha}^{*}(s)$ ) case, separately. Using the decomposition of the Nehari manifold as $\lambda$ varies, introduced by Tarantello [24], we will prove that the problem has at least two positive solutions for $\lambda$ sufficiently small.

We first prove the following theorem:

Theorem 1.1. Let $0<\alpha<2$ and $0 \leq s<\alpha<n$. Suppose $1<q<2<p<2_{\alpha}^{*}(s)$ and $\gamma<\gamma_{H}(\alpha)$. Then, there exits $\Lambda>0$ such that problem (11) has at least two positive solutions for any $\lambda \in(0, \Lambda)$.

The critical case is more challenging and requires information about the asymptotic behaviour of solutions of the following limiting problem at zero and infinity:

$$
\left\{\begin{aligned}
(-\Delta)^{\frac{\alpha}{2}} u-\gamma \frac{u}{|x|^{\alpha}} & =\frac{u^{2_{\alpha}^{*}(s)-1}}{|x|^{s}} & & \text { in } \mathbb{R}^{n} \\
u & \geq 0 & & \text { in } \mathbb{R}^{n},
\end{aligned}\right.
$$

where $0<\alpha<2,0 \leq s<\alpha, 2_{\alpha}^{*}(s)=\frac{2(n-s)}{n-\alpha}, 0 \leq \gamma<\gamma_{H}(\alpha)=2^{\alpha} \frac{\Gamma^{2}\left(\frac{n+\alpha}{4}\right)}{\Gamma^{2}\left(\frac{n-\alpha}{4}\right)}$. We get around the difficulty by working with certain asymptotic estimates for solutions of (2) recently obtained by the author and et al. in [14]; see Theorem [5.1. In order to use the results of [14, we may assume $g(x) \equiv 1$. Problem (11) therefore can be written as follows:

$$
\left\{\begin{array}{rlrl}
(-\Delta)^{\frac{\alpha}{2}} u-\gamma \frac{u}{|x|^{\alpha}} & =\lambda f(x)|u|^{q-2} u+\frac{|u|^{2_{\alpha}^{*}(s)-2} u}{|x|^{s}} & & \text { in } \Omega \\
u & =0 & \text { in } \mathbb{R}^{n} \backslash \Omega .
\end{array}\right.
$$

We then establish the following:

Theorem 1.2. Let $0<\alpha<2$ and $0 \leq s<\alpha<n$. Suppose $1<q<2<p=2_{\alpha}^{*}(s)$ and $0 \leq \gamma<\gamma_{H}(\alpha)$. Then, there exits $\Lambda^{*}>0$ such that problem (3) has at least two positive solutions for any $\lambda \in\left(0, \Lambda^{*}\right)$.

\section{$2 \quad$ Functional Setting}

We start by recalling and introducing suitable function spaces for the variational principles that will be needed in the sequel. We first recall that the non-local operator $(-\Delta)^{\frac{\alpha}{2}}$ is defined as

$$
(-\Delta)^{\frac{\alpha}{2}} u(x)=c(n, \alpha) P . V . \int_{\mathbb{R}^{n}} \frac{u(x)-u(y)}{|x-y|^{n+\alpha}} d y \quad \text { for } x \in \mathbb{R}^{n}, \quad \text { where } c(n, \alpha)=\frac{2^{\alpha-1} \Gamma\left(\frac{n+\alpha}{2}\right)}{\pi^{\frac{n}{2}}\left|\Gamma\left(-\frac{\alpha}{2}\right)\right|} .
$$

We denote by $H^{\frac{\alpha}{2}}\left(\mathbb{R}^{n}\right)$ the classical fractional Sobolev space endowed with the so-called Gagliardo norm 


$$
\|u\|_{H^{\frac{\alpha}{2}\left(\mathbb{R}^{n}\right)}}=\|u\|_{L^{2}\left(\mathbb{R}^{n}\right)}+\left(\int_{\mathbb{R}^{n}} \int_{\mathbb{R}^{n}} \frac{|u(x)-u(y)|^{2}}{|x-y|^{n+\alpha}} d x d y\right)^{\frac{1}{2}} .
$$

For $\alpha \in(0,2)$, the fractional Sobolev space $H_{0}^{\frac{\alpha}{2}}\left(\mathbb{R}^{n}\right)$ is defined as the completion of $C_{c}^{\infty}\left(\mathbb{R}^{n}\right)$ under the norm

$$
\|u\|_{H_{0}^{\frac{\alpha}{2}\left(\mathbb{R}^{n}\right)}}^{2}=\int_{\mathbb{R}^{n}}|2 \pi \xi|^{\alpha}|\mathcal{F} u(\xi)|^{2} d \xi=\int_{\mathbb{R}^{n}}\left|(-\Delta)^{\frac{\alpha}{4}} u\right|^{2} d x
$$

Let now $\Omega \subset \mathbb{R}^{n}$ be a smooth bounded domain. We define the space $X_{0}^{\frac{\alpha}{2}}(\Omega)$ as

$$
X_{0}^{\frac{\alpha}{2}}(\Omega)=\left\{u \in H^{\frac{\alpha}{2}}\left(\mathbb{R}^{n}\right): u=0 \text { a.e. in } \mathbb{R}^{n} \backslash \Omega\right\},
$$

and consider the following norm in $X_{0}^{\frac{\alpha}{2}}(\Omega)$ :

$$
\|u\|_{X_{0}^{\frac{\alpha}{2}}(\Omega)}=\left(\int_{\mathbb{R}^{n}} \int_{\mathbb{R}^{n}} \frac{|u(x)-u(y)|^{2}}{|x-y|^{n+\alpha}} d x d y\right)^{\frac{1}{2}} .
$$

We recall that $\left(X_{0}^{\frac{\alpha}{2}}(\Omega),\|\cdot\|_{X_{0}^{\frac{\alpha}{2}}(\Omega)}\right)$ is a Hilbert space with the scalar product

$$
\langle u, v\rangle_{X_{0}^{\frac{\alpha}{2}}(\Omega)}=\int_{\mathbb{R}^{n}} \int_{\mathbb{R}^{n}} \frac{(u(x)-u(y))(v(x)-v(y))}{|x-y|^{n+\alpha}} d x d y .
$$

Remark 2.1. It was shown in [5] that the sub-space $C_{0}^{\infty}(\Omega)$ is dense in $X_{0}^{\frac{\alpha}{2}}(\Omega)$. So, we can consider $X_{0}^{\frac{\alpha}{2}}(\Omega)$ as the completion of $C_{0}^{\infty}(\Omega)$ with with respect to the norm $\|\cdot\|_{X_{0}^{\frac{\alpha}{2}}(\Omega)}$.

Definition 2.2. We say $u \in X_{0}^{\frac{\alpha}{2}}(\Omega)$ is a weak solution of (1) , if for every $\phi \in X_{0}^{\frac{\alpha}{2}}(\Omega)$, we have

$$
c(n, \alpha)\langle u, \phi\rangle_{X_{0}^{\frac{\alpha}{2}}(\Omega)}-\gamma \int_{\Omega} \frac{u \phi}{|x|^{\alpha}} d x=\lambda \int_{\Omega} f(x)|u|^{q-1} \phi d x-\int_{\Omega} g(x) \frac{|u|^{p-1} \phi}{|x|^{s}} d x .
$$

The energy functional corresponding to (11) is

$$
I_{\lambda, p}(u)=\frac{c(n, \alpha)}{2}\|u\|_{X_{0}^{\alpha}(\Omega)}^{2}-\frac{\gamma}{2} \int_{\Omega} \frac{|u|^{2}}{|x|^{\alpha}} d x-\frac{\lambda}{q} \int_{\Omega} f(x)|u|^{q} d x-\frac{1}{p} \int_{\Omega} g(x) \frac{|u|^{p}}{|x|^{s}} d x .
$$

Recall that any critical point $u$ of $I_{\lambda, p}(u)$ is a weak solution for (1). The starting point of the study of existence of weak solutions to problem (11) is therefore the following fractional inequalities which will guarantee that the above functional is well defined and bounded below on the right function spaces.

We start with the fractional Sobolev inequality [10, which asserts that for $n>\alpha$ and $0<\alpha<2$, there exists a constant $S(n, \alpha)>0$ such that

$$
\|u\|_{L^{2 *}\left(\mathbb{R}^{n}\right)}^{2} \leq S(n, \alpha) \int_{\mathbb{R}^{n}} \int_{\mathbb{R}^{n}} \frac{|u(x)-u(y)|^{2}}{|x-y|^{n+\alpha}} d x d y \quad \text { for } u \in H^{\frac{\alpha}{2}}\left(\mathbb{R}^{n}\right),
$$

where $2_{\alpha}^{*}=\frac{2 n}{n-\alpha}$. Another important inequality is the fractional Hardy inequality [16, which states that under the same conditions on $n$ and $\alpha$, there exists a constant $\gamma(n, \alpha)>0$ such that

$$
\gamma_{H}(\alpha) \int_{\mathbb{R}^{n}} \frac{|u|^{2}}{|x|^{\alpha}} d x \leq \int_{\mathbb{R}^{n}}|\xi|^{\alpha}|\mathcal{F} u(\xi)|^{2} d \xi \quad \text { for } u \in C_{0}^{\infty}\left(\mathbb{R}^{n}\right),
$$

where $\mathcal{F} u(x)=\frac{1}{(2 \pi)^{\frac{n}{2}}} \int_{\mathbb{R}^{n}} e^{-i \xi x} u(x) d x$ is the Fourier transform of $u$. It was shown in [16] that $\gamma_{H}(\alpha):=2^{\alpha} \frac{\Gamma^{2}\left(\frac{n+\alpha}{4}\right)}{\Gamma^{2}\left(\frac{n-\alpha}{4}\right)}$ is the best constant in the above fractional Hardy inequality. Note that $\gamma_{H}(\alpha)$ converges to the best classical Hardy constant $\frac{(n-2)^{2}}{4}$ when $\alpha \rightarrow 2$. 
By Proposition 3.6 in [11, for any $u \in H^{\frac{\alpha}{2}}\left(\mathbb{R}^{n}\right)$, we have the following relation between the fractional Laplacian operator $(-\Delta)^{\frac{\alpha}{2}}$ and the fractional Sobolev space $H^{\frac{\alpha}{2}}\left(\mathbb{R}^{n}\right)$ :

$$
\int_{\mathbb{R}^{n}}|\xi|^{\alpha}|\mathcal{F} u(\xi)|^{2} d \xi=c(n, \alpha) \int_{\mathbb{R}^{n}} \int_{\mathbb{R}^{n}} \frac{|u(x)-u(y)|^{2}}{|x-y|^{n+\alpha}} d x d y .
$$

The fractional Hard inequality then can be written as

$$
\gamma_{H}(\alpha) \int_{\mathbb{R}^{n}} \frac{|u|^{2}}{|x|^{\alpha}} d x \leq c(n, \alpha) \int_{\mathbb{R}^{n}} \int_{\mathbb{R}^{n}} \frac{|u(x)-u(y)|^{2}}{|x-y|^{n+\alpha}} d x d y \quad \text { for } u \in C_{0}^{\infty}\left(\mathbb{R}^{n}\right) .
$$

By interpolating these inequalities via Hölder's inequalities, one gets the following fractional HardySobolev inequality.

Lemma 2.3 (Lemma 2.1 in [13]). Assume that $0<\alpha<2,0 \leq s \leq \alpha<n$ and $2<p \leq 2_{\alpha}^{*}(s)=$ $\frac{2(n-s)}{n-\alpha}$. Then, there exists a positive constant $C$ such that

$$
C\left(\int_{\Omega} \frac{|u|^{p}}{|x|^{s}} d x\right)^{\frac{2}{p}} \leq c(n, \alpha) \int_{\mathbb{R}^{n}} \int_{\mathbb{R}^{n}} \frac{|u(x)-u(y)|^{2}}{|x-y|^{n+\alpha}} d x d y-\gamma \int_{\Omega} \frac{|u|^{2}}{|x|^{\alpha}} d x \quad \text { for } u \in X_{0}^{\frac{\alpha}{2}}(\Omega),
$$

as long as $\gamma<\gamma_{H}(\alpha):=2^{\alpha} \frac{\Gamma^{2}\left(\frac{n+\alpha}{4}\right)}{\Gamma^{2}\left(\frac{n-\alpha}{4}\right)}$.

Finally, we can define the general best Hardy-sobolev constant in the above inequality as

$$
S_{p}:=\inf _{u \in X_{0}^{\frac{\alpha}{2}}(\Omega) \backslash\{0\}} \frac{c(n, \alpha) \int_{\mathbb{R}^{n}} \int_{\mathbb{R}^{n}} \frac{|u(x)-u(y)|^{2}}{|x-y|^{n+\alpha}} d x d y-\gamma \int_{\Omega} \frac{|u|^{2}}{|x|^{\alpha}} d x}{\left(\int_{\Omega} \frac{\left.u\right|^{p}}{|x|^{s}} d x\right)^{\frac{2}{p}}},
$$

where $2<p \leq 2_{\alpha}^{*}(s)=\frac{2(n-s)}{n-\alpha}$, and $\gamma<\gamma_{H}(\alpha)$.

Note that the frational Hardy inequality (7) asserts that $X_{0}^{\frac{\alpha}{2}}(\Omega)$ is embedded in the weighted space $L^{2}\left(\Omega,|x|^{-\alpha}\right)$ and that this embeding is continuous. If $\gamma<\gamma_{H}(\alpha)$, it follows from the fractional Hardy inequality (7) that

$$
\|u\|:=\left(c(n, \alpha) \int_{\mathbb{R}^{n}} \int_{\mathbb{R}^{n}} \frac{|u(x)-u(y)|^{2}}{|x-y|^{n+\alpha}} d x d y-\gamma \int_{\Omega} \frac{|u|^{2}}{|x|^{\alpha}} d x\right)^{\frac{1}{2}}
$$

is well-defined on $X_{0}^{\frac{\alpha}{2}}(\Omega)$. It also is equivalent to the norm $\|\cdot\|_{X_{0}^{\frac{\alpha}{2}}(\Omega)}$. Thus, we can rewrite the functional $I_{\lambda, p}$ as

$$
I_{\lambda, p}(u)=\frac{1}{2}\|u\|^{2}-\frac{\lambda}{q} \int_{\Omega} f(x)|u|^{q} d x-\frac{1}{p} \int_{\Omega} g(x) \frac{|u|^{p}}{|x|^{s}} d x .
$$

\section{Preliminary Results}

For $\lambda>0$, we will consider the following Nehari minimization problem:

$$
\mathcal{M}=\inf \left\{I_{\lambda, p}(u): u \in \mathcal{N}\right\}, \quad \text { where } \mathcal{N}=\left\{u \in X_{0}^{\frac{\alpha}{2}}(\Omega):\left\langle I_{\lambda, p}^{\prime}(u), u\right\rangle=0\right\} .
$$

Define

$$
\phi_{\lambda, p}(u):=\left\langle I_{\lambda, p}^{\prime}(u), u\right\rangle=\|u\|^{2}-\lambda \int_{\Omega} f(x)|u|^{q} d x-\int_{\Omega} g(x) \frac{|u|^{p}}{|x|^{s}} d x .
$$

So, 


$$
\left\langle\phi_{\lambda, p}^{\prime}(u), u\right\rangle=2\|u\|^{2}-\lambda q \int_{\Omega} f(x)|u|^{q} d x-p \int_{\Omega} g(x) \frac{|u|^{p}}{|x|^{s}} d x .
$$

Thus, for all $u \in \mathcal{N}$, we have the following identities which will be used frequently in this paper.

$$
\begin{gathered}
\|u\|^{2}=\lambda \int_{\Omega} f(x)|u|^{q} d x+\int_{\Omega} g(x) \frac{|u|^{p}}{|x|^{s}} d x, \\
I_{\lambda, p}(u)=\left(\frac{1}{2}-\frac{1}{q}\right)\|u\|^{2}-\left(\frac{1}{p}-\frac{1}{q}\right) \int_{\Omega} g(x) \frac{|u|^{p}}{|x|^{s}} d x \\
=\left(\frac{1}{2}-\frac{1}{p}\right)\|u\|^{2}+\left(\frac{1}{p}-\frac{1}{q}\right) \lambda \int_{\Omega} f(x)|u|^{q} d x,
\end{gathered}
$$

and

$$
\begin{aligned}
\left\langle\phi_{\lambda, p}^{\prime}(u), u\right\rangle & =(2-q)\|u\|^{2}-(p-q) \int_{\Omega} g(x) \frac{|u|^{p}}{|x|^{s}} d x \\
& =(2-p)\|u\|^{2}-\lambda(q-p) \int_{\Omega} f(x)|u|^{q} d x .
\end{aligned}
$$

Now we split $\mathcal{N}$ into three parts:

$$
\begin{aligned}
& \mathcal{N}^{+}=\left\{u \in \mathcal{N}:\left\langle\phi_{\lambda, p}^{\prime}(u), u\right\rangle>0\right\}, \\
& \mathcal{N}^{0}=\left\{u \in \mathcal{N}:\left\langle\phi_{\lambda, p}^{\prime}(u), u\right\rangle=0\right\}, \\
& \mathcal{N}^{-}=\left\{u \in \mathcal{N}:\left\langle\phi_{\lambda, p}^{\prime}(u), u\right\rangle<0\right\} .
\end{aligned}
$$

We first show that for $\lambda$ small enough, $\mathcal{N}^{0}$ is an empty set.

Lemma 3.1. There exists a constant $\Lambda_{1}:=\Lambda_{1}(p)>0$ such that for any $\lambda \in\left(0, \Lambda_{1}\right)$, we have $\mathcal{N}^{0}=\emptyset$.

Proof. We deduce by contradiction. Suppose that there exists $u \in X_{0}^{\frac{\alpha}{2}}(\Omega) \backslash\{0\}$ such that $u \in \mathcal{N}^{0}$, that is, $\left\langle\phi^{\prime}(u), u\right\rangle=0$. We will consider the two following cases:

Case 1: $\int_{\Omega} f(x)|u|^{q} d x=0$. Using (13) and the fact that $\int_{\Omega} f(x)|u|^{q} d x=0$, we get

$$
0=\left\langle\phi_{\lambda, p}^{\prime}(u), u\right\rangle=(2-p)\|u\|^{2} .
$$

On the other hand, the assumption $p>2$ implies that $(2-p)\|u\|^{2}<0$, which contradicts the last equality.

Case 2: $\int_{\Omega} f(x)|u|^{q} d x \neq 0$. It follows from (13) that

$$
\|u\|^{2}=\lambda\left(\frac{p-q}{p-2}\right) \int_{\Omega} f(x)|u|^{q} d x .
$$

By the definition of $S_{p}$ and the Hölder inequality, we get that

$$
\|u\|^{2} \leq \lambda\left(\frac{p-q}{p-2}\right) A\left(\int_{\Omega} \frac{|u|^{p}}{|x|^{s}} d x\right)^{\frac{q}{p}} \leq \lambda\left(\frac{p-q}{p-2}\right) A S_{p}^{-\frac{q}{2}}\|u\|^{q},
$$

which yields

$$
\|u\| \leq\left(\lambda\left(\frac{p-q}{p-2}\right) A S_{p}^{-\frac{q}{2}}\right)^{\frac{1}{2-q}}
$$


where $A:=\left(\int_{\Omega}|x|^{\frac{s q}{p-q}}|f(x)|^{\frac{p}{p-q}} d x\right)^{\frac{p-q}{p}}$. Define the functional $J_{\lambda, p}: \mathcal{N} \longrightarrow \mathbb{R}$ as

$$
J_{\lambda, p}(u)=\left(\frac{p-2}{2-q}\right)\left(\frac{2-q}{p-q}\right)^{\frac{p-1}{p-2}}\left(\frac{\|u\|^{2(p-1)}}{\int_{\Omega} g(x) \frac{|u|^{p}}{\left.x\right|^{s}} d x}\right)^{\frac{1}{p-2}}-\lambda \int_{\Omega} f(x)|u|^{q} d x .
$$

We claim that $J_{\lambda}(u)=0$ for all $u \in \mathcal{N}^{0}$. Indeed, by (13), we have

$$
\int_{\Omega} g(x) \frac{|u|^{p}}{|x|^{s}} d x=\left(\frac{2-q}{p-q}\right)\|u\|^{2} \quad \text { and } \quad \lambda \int_{\Omega} f(x)|u|^{q} d x=\left(\frac{p-2}{2-q}\right)\|u\|^{2} \quad \text { for all } u \in \mathcal{N}^{0} .
$$

Thus,

$$
\begin{aligned}
J_{\lambda, p}(u) & =\left(\frac{p-2}{2-q}\right)\left(\frac{2-q}{p-q}\right)^{\frac{p-1}{p-2}}\left(\frac{\|u\|^{2(p-1)}}{\left(\frac{2-q}{p-q}\right)\|u\|^{2}}\right)^{\frac{1}{p-2}}-\lambda \int_{\Omega} f(x)|u|^{q} d x \\
& =\left(\frac{p-2}{2-q}\right)\|u\|^{2}-\lambda \int_{\Omega} f(x)|u|^{q} d x=0 \quad \text { for all } u \in \mathcal{N}^{0} .
\end{aligned}
$$

Let $C(p, q):=\left(\frac{p-2}{2-q}\right)\left(\frac{2-q}{p-q}\right)^{\frac{p-1}{p-2}}$. By Hölder's inequality and the definition of $S_{p}$, we obtain

$$
\begin{aligned}
J_{\lambda, p}(u) & \geq C(p, q)\left(\frac{\|u\|^{2(p-1)}}{\int_{\Omega} g(x) \frac{|u|^{p}}{|x|^{s}} d x}\right)^{\frac{1}{p-2}}-\lambda A\left(\int_{\Omega} \frac{|u|^{p}}{|x|^{s}} d x\right)^{\frac{q}{p}} \\
& \geq\left(\int_{\Omega} \frac{|u|^{p}}{|x|^{s}} d x\right)^{\frac{q}{p}}\left[C(p, q) S_{p}^{\frac{p-1}{p-2}}\|g\|_{\infty}^{\frac{1}{2-p}}\left(\int_{\Omega} \frac{|u|^{p}}{|x|^{s}} d x\right)^{\frac{1-q}{p}}-\lambda A\right] \\
& \geq\left(\int_{\Omega} \frac{|u|^{p}}{|x|^{s}} d x\right)^{\frac{q}{p}}\left[C(p, q) S_{p}^{\frac{p-1}{p-2}+\frac{q-1}{2-q}}\left(\frac{\lambda A(p-q)}{p-2}\right)^{\frac{1-q}{2-q}}\|g\|_{\infty}^{\frac{1}{2-p}}-\lambda A\right] .
\end{aligned}
$$

Thus, we get that $J_{\lambda, p}(u)>0$ for $\lambda$ sufficiently small. Therefore, there exists $\Lambda_{1}:=\Lambda_{1}(p)>0$ such that $J_{\lambda, p}(u)>0$ for all $\lambda \in\left(0, \Lambda_{1}\right)$ and $u \in \mathcal{N}^{0}$. This contradicts (18) and completes the proof.

Lemma 3.2. If $u \in \mathcal{N}^{+} \backslash\{0\}$, then $\int_{\Omega} f(x)|u|^{q} d x>0$.

Proof. Since $u \in \mathcal{N}^{+} \backslash\{0\}$, we have $\left\langle\phi_{\lambda, p}^{\prime}(u), u\right\rangle>0$. It then follows from (13) that

$$
\left(\frac{2-q}{p-q}\right)\|u\|^{2}>\int_{\Omega} g(x) \frac{|u|^{p}}{|x|^{s}} d x .
$$

By (11) and the last inequality, we get that

$$
\begin{aligned}
\lambda \int_{\Omega} f(x)|u|^{q} d x & =\|u\|^{2}-\int_{\Omega} g(x) \frac{|u|^{p}}{|x|^{s}} d x \\
& >\|u\|^{2}-\left(\frac{2-q}{p-q}\right)\|u\|^{2} \\
& >\left(\frac{p-2}{p-q}\right)\|u\|^{2}>0 .
\end{aligned}
$$


From Lemma 3.1, we deduce that $\mathcal{N}=\mathcal{N}^{+} \cup \mathcal{N}^{-}$for any $\lambda \in\left(0, \Lambda_{1}\right)$. Define

$$
\mathcal{M}^{+}:=\inf _{\mathcal{N}^{+}} I_{\lambda, p}(u) \quad \text { and } \quad \mathcal{M}^{-}:=\inf _{\mathcal{N}^{-}} I_{\lambda, p}(u)
$$

Lemma 3.3. For any $\lambda \in\left(0, \Lambda_{1}\right)$, the minimizers on $\mathcal{N}$ are critical points for $I_{\lambda, p}$ in $\left(X_{0}^{\frac{\alpha}{2}}(\Omega)\right)^{\prime}$, where $\left(X_{0}^{\frac{\alpha}{2}}(\Omega)\right)^{\prime}$ is dual space of $X_{0}^{\frac{\alpha}{2}}(\Omega)$.

Proof. Suppose that $\bar{u}$ is a local minimum for $I_{\lambda, p}$. Thus, it satisfies the following minimization problem:

$$
\min _{u \in X_{0}^{\frac{\alpha}{2}}(\Omega)}\left\{I_{\lambda, p}(u): \phi_{\lambda, p}=\left\langle I_{\lambda, p}^{\prime}(u), u\right\rangle=0\right\}
$$

which gives

$$
I_{\lambda, p}(\bar{u})=\min _{u \in X_{0}^{\frac{\alpha}{2}}(\Omega)} I_{\lambda, p}(u) \quad \text { and } \quad \phi_{\lambda, p}(\bar{u})=\left\langle I_{\lambda, p}^{\prime}(\bar{u}), \bar{u}\right\rangle=0 .
$$

It follows from the theorem of Lagrange multiplies that there exists $\theta$ such that $I_{\lambda, p}^{\prime}(\bar{u})=\theta \phi_{\lambda, p}^{\prime}(\bar{u})$ in $\left(X_{0}^{\frac{\alpha}{2}}(\Omega)\right)^{\prime}$. So, we have

$$
0=\left\langle I_{\lambda, p}^{\prime}(\bar{u}), \bar{u}\right\rangle=\left\langle\theta \phi_{\lambda, p}^{\prime}(\bar{u}), u\right\rangle=\theta\left\langle\phi_{\lambda, p}^{\prime}(\bar{u}), \bar{u}\right\rangle
$$

Thus,

$$
\text { either } \theta=0 \quad \text { or } \quad\left\langle\phi_{\lambda, p}^{\prime}(\bar{u}), \bar{u}\right\rangle=0 \text {. }
$$

By Lemma 3.1 we get that $\left\langle\phi_{\lambda, p}^{\prime}(u), u\right\rangle \neq 0$ for $u \neq 0$. Therefore $\theta=0$. Thus, we obtain $I_{\lambda, p}^{\prime}(\bar{u})=$ $\theta \phi_{\lambda, p}^{\prime}(\bar{u})=0$ in $\left(X_{0}^{\frac{\alpha}{2}}(\Omega)\right)^{\prime}$, that is, $\bar{u}$ is a critical point for $I_{\lambda, p}$ in $\left(X_{0}^{\frac{\alpha}{2}}(\Omega)\right)^{\prime}$.

Lemma 3.4. Let $\Lambda_{2}:=\Lambda_{2}(p)=\left(\frac{p-2}{p-q}\right)\left(\frac{2-q}{p-q}\right)^{\frac{2-q}{p-2}} S_{p}^{\frac{p-q}{p-2}} A^{-1}\|g\|_{\infty}^{\frac{q-2}{p-2}}$. Then, for all $u \in X_{0}^{\frac{\alpha}{2}}(\Omega) \backslash\{0\}$ and $\lambda \in\left(0, \Lambda_{2}\right)$, there exist unique $t^{+}(u)$ and $t^{-}(u)$ such that

1. $0 \leq t^{+}(u)<t_{\max }<t^{-}(u)$.

2. $t^{-}(u) u \in \mathcal{N}^{-}$and $t^{+}(u) u \in \mathcal{N}^{+}$.

3. $I_{\lambda, p}\left(t^{-}(u) u\right)=\max _{t>t_{\max }} I_{\lambda, p}(t u)$ and $I_{\lambda, p}\left(t^{+}(u) u\right)=\min _{0 \leq t \leq t^{-}(u)} I_{\lambda, p}(t u)$.

4. $\mathcal{N}^{-}=\left\{u \in X_{0}^{\frac{\alpha}{2}}(\Omega) \backslash\{0\}: t^{-}\left(\frac{u}{\|u\|}\right)=\|u\|\right\}$,

where $t_{\max }:=\left(\frac{2-q}{p-q} \frac{\|u\|^{2}}{\int_{\Omega} g(x) \frac{\left.u\right|^{p}}{|x|^{s}} d x}\right)^{\frac{1}{p-2}}$. Moreover, $t^{+}(u)>0$ if and only if $\int_{\Omega} f(x)|u|^{q} d x>0$.

Proof. For $t \geq 0$, define

$$
h(t)=t^{2-q}\|u\|^{2}-t^{p-q} \int_{\Omega} g(x) \frac{|u|^{p}}{|x|^{s}} d x .
$$

Straightforuard computations yield that $h(0)=0, \lim _{t \rightarrow \infty} h(t)=-\infty, h^{\prime}\left(t_{\max }\right)=0$, and $h(t)$ is attained its maximum at $t_{\max }$. In addition, $h(t)$ is increasing for $t \in\left[0, t_{\max }\right)$ and decreasing for $t \in\left(t_{\max }, \infty\right)$. So, we have 


$$
h\left(t_{\max }\right)=\left(\frac{p-2}{p-q}\right)\left(\frac{2-q}{p-q}\right)^{\frac{2-q}{p-2}}\|u\|^{q}\left(\frac{\|u\|^{p}}{\int_{\Omega} g(x) \frac{|u|^{p}}{|x|^{s}} d x}\right)^{\frac{2-q}{p-2}} .
$$

By Hölder's inequality and the definition of $S_{p}$, we obtain

$$
h\left(t_{\max }\right) \geq\left(\frac{p-2}{p-q}\right)\left(\frac{2-q}{p-q}\right)^{\frac{2-q}{p-2}}\|u\|^{q}\|g\|_{\infty}^{\frac{q-2}{p-2}} S_{p}^{\frac{p(2-q)}{2(p-2)}} .
$$

We will now consider the two following cases:

Case 1: $\int_{\Omega} f(x)|u|^{q} d x \leq 0$. In this case, there exists a unique $t^{-}:=t^{-}(u)>t_{\max }$ such that

$$
h\left(t^{-}\right)=\lambda \int_{\Omega} f(x)|u|^{q} d x \text { and } h^{\prime}\left(t^{-}\right)<0 .
$$

We claim that $t^{-} u \in \mathcal{N}^{-}$. Indeed, clearly $t^{-} u \in X_{0}^{\frac{\alpha}{2}}(\Omega)$, and (21) implies that

$$
\begin{aligned}
\left\langle I_{\lambda, p}^{\prime}\left(t^{-} u\right), t^{-} u\right\rangle & =\left\|t^{-} u\right\|^{2}-\lambda \int_{\Omega} f(x)\left|t^{-} u\right|^{q} d x-\int_{\Omega} g(x) \frac{\left|t^{-} u\right|^{p}}{|x|^{s}} d x \\
& =\left(t^{-}\right)^{q}\left(\left(t^{-}\right)^{2-q}\|u\|^{2}-\left(t^{-}\right)^{p-q} \int_{\Omega} g(x) \frac{|u|^{p}}{|x|^{s}} d x-\lambda \int_{\Omega} f(x)|u|^{q} d x\right) \\
& =\left(t^{-}\right)^{q}\left(h\left(t^{-}\right)-\lambda \int_{\Omega} f(x)|u|^{q} d x\right)=0,
\end{aligned}
$$

and

$$
\begin{aligned}
\left\langle\phi_{\lambda, p}^{\prime}\left(t^{-} u\right), t^{-} u\right\rangle & =2\left\|t^{-} u\right\|^{2}-\lambda q \int_{\Omega} f(x)\left|t^{-} u\right|^{q} d x-p \int_{\Omega} g(x) \frac{\left|t^{-} u\right|^{p}}{|x|^{s}} d x \\
& =(2-q)\left\|t^{-} u\right\|^{2}-(p-q) \int_{\Omega} g(x) \frac{\left|t^{-} u\right|^{p}}{|x|^{s}} d x \\
& =\left(t^{-}\right)^{q+1}\left((2-q)\left(t^{-}\right)^{2-q-1}\|u\|^{2}-(p-q)\left(t^{-}\right)^{p-q-1} \int_{\Omega} g(x) \frac{|u|^{p}}{|x|^{s}} d x\right) \\
& =\left(t^{-}\right)^{q+1} h^{\prime}\left(t^{-}\right)<0 .
\end{aligned}
$$

This proves the claim, and we have that $t^{-} u \in \mathcal{N}^{-}$. In order to prove that $I_{\lambda, p}\left(t^{-} u\right)=\max _{t \geq t_{\max }} I_{\lambda, p}(t u)$, we need to show that

$$
\frac{d}{d t} I_{\lambda, p}\left(t^{-} u\right)=0 \text { and } \frac{d^{2}}{d t^{2}} I_{\lambda, p}(t u)<0 \quad \text { for } t>t_{\max } .
$$

It follows from (21) that

$$
\begin{aligned}
\frac{d}{d t} I_{\lambda, p}\left(t^{-} u\right) & =t^{-}\|u\|^{2}-\lambda\left(t^{-}\right)^{q-1} \int_{\Omega} f(x)|u|^{q} d x-\left(t^{-}\right)^{p-1} \int_{\Omega} g(x) \frac{|u|^{p}}{|x|^{s}} d x \\
& =\left(t^{-}\right)^{q-1}\left(h\left(t^{-}\right)-\lambda \int_{\Omega} f(x)|u|^{q} d x\right)=0 .
\end{aligned}
$$

We also have 


$$
\begin{aligned}
t^{2} \frac{d^{2}}{d t^{2}} I_{\lambda, p}(t u) & =\left\|t^{-} u\right\|^{2}-\lambda(q-1) \int_{\Omega} f(x)\left|t^{-} u\right|^{q} d x-(p-1) \int_{\Omega} g(x) \frac{\left|t^{-} u\right|^{p}}{|x|^{s}} d x \\
& =\left(t^{-}\right)^{q+1}\left((2-q)\left(t^{-}\right)^{2-q-1}\|u\|^{2}-(p-q)\left(t^{-}\right)^{p-q-1} \int_{\Omega} g(x) \frac{|u|^{p}}{|x|^{s}} d x\right) \\
& =\left(t^{-}\right)^{q+1} h^{\prime}\left(t^{-}\right)<0 \quad \text { for all } t>t_{\max } .
\end{aligned}
$$

Case 2: $\int_{\Omega} f(x)|u|^{q} d x>0$. Using Hölder's inequality and (20), we have

$$
\begin{aligned}
0=h(0) & <\int_{\Omega} f(x)|u|^{q} d x \leq \lambda A S_{p}^{-\frac{q}{2}}\|u\|^{q} \\
& \leq\left(\frac{p-2}{p-q}\right)\left(\frac{2-q}{p-q}\right)^{\frac{2-q}{p-2}}\|g\|_{\infty}^{\frac{q-2}{p-2}} S_{p}^{\frac{p(2-q)}{2(p-2)}}\|u\|^{q} \\
& \leq h\left(t_{\max }\right) \quad \text { for } 0<\lambda<\Lambda_{2} .
\end{aligned}
$$

Using the assumption $\int_{\Omega} f(x)|u|^{q} d x>0$ and the fact that $h\left(t_{\max }\right)>0$, we get that there exist unique $t^{+}:=t^{+}(u)$ and $t^{-}:=t^{-}(u)$ such that $t^{+}<t_{\max }<t^{-}$, and

$$
h\left(t^{-}\right)=\lambda \int_{\Omega} f(x)|u|^{q} d x=h\left(t^{+}\right) \text {and } h^{\prime}\left(t^{-}\right)<0<h^{\prime}\left(t^{+}\right) .
$$

Lemma 3.5. The following hold.

1. $\mathcal{M} \leq \mathcal{M}^{+}<0$.

2. Let $\Lambda_{3}:=\Lambda_{3}(p)=\frac{p-2}{p-q}$. Then, the functional $I_{\lambda, p}$ is coercive and bounded below on $\mathcal{N}$ for any $\lambda \in\left(0, \Lambda_{3}\right]$.

Proof. By (12), for any $u \in \mathcal{N}$, we have

$$
I_{\lambda, p}(u)=\left(\frac{1}{2}-\frac{1}{p}\right)\|u\|^{2}+\left(\frac{1}{p}-\frac{1}{q}\right) \lambda \int_{\Omega} f(x)|u|^{q} d x .
$$

1. Suppose that $u \in \mathcal{N}^{+}$. It follows from (13) that

$$
\begin{aligned}
I_{\lambda, p}(u) & <\left(\frac{p-2}{2 p}\right)\left(\frac{p-q}{p-2}\right) \lambda \int_{\Omega} f(x)|u|^{q} d x+\left(\frac{q-p}{p q}\right) \lambda \int_{\Omega} f(x)|u|^{q} d x \\
& =-\frac{(p-q)(2-q)}{2 p q} \lambda \int_{\Omega} f(x)|u|^{q} d x .
\end{aligned}
$$

By Lemma 3.2, we have $\int_{\Omega} f(x)|u|^{q} d x>0$. Thus,

$$
I_{\lambda, p}(u)<-\frac{(p-q)(2-q)}{2 p q} \lambda \int_{\Omega} f(x)|u|^{q} d x<0,
$$

which yields

$$
\mathcal{M} \leq \mathcal{M}^{+}<0 .
$$

2. Using Hölder and Young's inequality, we get that 


$$
I_{\lambda, p}(u) \geq \frac{1}{2 p}((p-2)-\lambda(p-q))\|u\|^{2}-\lambda\left(\frac{p-q}{p q}\right)\left(\frac{2-q}{2}\right)\left(A S_{p}^{-\frac{q}{2}}\right)^{\frac{2}{2-q}} .
$$

Since $0<\lambda<\frac{p-2}{p-q}$, the functional $I_{\lambda, p}$ is coercive and bounded below on $\mathcal{N}$, and we have

$$
I_{\lambda, p}(u) \geq-\lambda\left(\frac{p-q}{p q}\right)\left(\frac{2-q}{2}\right)\left(A S_{p}^{-\frac{q}{2}}\right)^{\frac{2}{2-q}} .
$$

Lemma 3.6. For each $u \in \mathcal{N} \backslash\{0\}$, there exist $\epsilon>0$ and a differentiable function $\sigma: B(0, \epsilon) \subset$ $X_{0}^{\frac{\alpha}{2}}(\Omega) \longrightarrow \mathbb{R}^{+}$such that $\sigma(0)=1, \sigma(v)(u-v) \in \mathcal{N}$, and

$$
\left\langle\sigma^{\prime}(0), v\right\rangle=\frac{2 c(n, \alpha)\langle u, v\rangle_{X_{0}^{\frac{\alpha}{2}}(\Omega)}-2 \gamma \int_{\Omega} \frac{u v}{|x|^{\alpha}} d x-q \lambda \int_{\Omega} f(x)|u|^{q-1} u v d x-p \int_{\Omega} g(x) \frac{|u|^{p-2} u v}{|x|^{s}} d x}{(2-q)\left(c(n, \alpha) \int_{\mathbb{R}^{n}} \int_{\mathbb{R}^{n}} \frac{|u(x)-u(y)|^{2}}{|x-y|^{n+\alpha}} d x d y-\gamma \int_{\Omega} \frac{|u|^{2}}{|x|^{\alpha}} d x\right)-(p-q) \int_{\Omega} g(x) \frac{|u|^{p}}{|x|^{s}} d x},
$$

for all $v \in X_{0}^{\frac{\alpha}{2}}(\Omega)$. Here $B(0, \epsilon):=\left\{u \in X_{0}^{\frac{\alpha}{2}}(\Omega):\|u\|<\epsilon\right\}$.

Proof. For $u \in \mathcal{N}$, define $G: \mathbb{R} \times X_{0}^{\frac{\alpha}{2}}(\Omega) \longrightarrow \mathbb{R}$ as

$$
G(t, v)=\left\langle I_{\lambda, p}^{\prime}(t(u-v)), t(u-v)\right\rangle .
$$

So, we have

$$
\begin{aligned}
G(t, v) & =t^{2} c(n, \alpha) \int_{\mathbb{R}^{n}} \int_{\mathbb{R}^{n}} \frac{|u(x)-u(y)|^{2}}{|x-y|^{n+\alpha}} d x d y-t^{2} \gamma \int_{\Omega} \frac{|u|^{2}}{|x|^{\alpha}} d x \\
& -\lambda t^{q} \int_{\Omega} f(x)|u-v|^{q} d x-t^{p} \int_{\Omega} g(x) \frac{|u|^{p}}{|x|^{s}} d x,
\end{aligned}
$$

and

$$
G(1,0)=\left\langle I_{\lambda, p}^{\prime}(u), u\right\rangle .
$$

By Lemma 3.1, we obtain $\frac{d}{d t} G(1,0) \neq 0$, that is,

$$
\begin{aligned}
0 \neq \frac{d}{d t} G(1,0) & =2 t c(n, \alpha) \int_{\mathbb{R}^{n}} \int_{\mathbb{R}^{n}} \frac{|u(x)-u(y)|^{2}}{|x-y|^{n+\alpha}} d x d y-2 t \gamma \int_{\Omega} \frac{|u|^{2}}{|x|^{\alpha}} d x \\
& -q \lambda t^{q-1} \int_{\Omega} f(x)|u|^{q} d x-\left.p t^{p-1} \int_{\Omega} g(x) \frac{|u|^{p}}{|x|^{s}} d x\right|_{t=1} \\
& =(2-q)\|u\|^{2}-(p-q) \int_{\Omega} g(x) \frac{|u|^{p}}{|x|^{s}} d x .
\end{aligned}
$$

According to the implicit function theorem, there exist $\epsilon>0$ and a differentiable function $\sigma$ : $B(0, \epsilon) \longrightarrow \mathbb{R}$ such that $\sigma(0)=1$, and

$$
\left\langle\sigma^{\prime}(0), v\right\rangle=\frac{2 c(n, \alpha)\langle u, v\rangle_{X_{0}^{\frac{\alpha}{2}}(\Omega)}-2 \gamma \int_{\Omega} \frac{u v}{|x|^{\alpha}} d x-q \lambda \int_{\Omega} f(x)|u|^{q-1} u v d x-p \int_{\Omega} g(x) \frac{|u|^{p-2} u v}{|x|^{s}} d x}{(2-q)\left(c(n, \alpha) \int_{\mathbb{R}^{n}} \int_{\mathbb{R}^{n}} \frac{|u(x)-u(y)|^{2}}{|x-y|^{n+\alpha}} d x d y-\gamma \int_{\Omega} \frac{|u|^{2}}{|x|^{\alpha}} d x\right)-(p-q) \int_{\Omega} g(x) \frac{|u|^{p}}{|x|^{s}} d x} .
$$

Moreover, we have $G(\sigma(v), v)=0$, for all $v \in B(0, \epsilon)$, which implies that $\left\langle I_{\lambda, p}^{\prime}(\sigma(v)(u-v)), \sigma(v)(u-\right.$ $v))\rangle=0$, that is, $\sigma(v)(u-v) \in \mathcal{N}$. 
Lemma 3.7. For each $u \in \mathcal{N}^{-} \backslash\{0\}$, there exist $\epsilon>o$ and a differentiable function $\sigma_{-}: B(0, \epsilon) \subset$ $X_{0}^{\frac{\alpha}{2}}(\Omega) \longrightarrow \mathbb{R}^{+}$such that $\sigma_{-}(0)=1, \sigma_{-}(v)(u-v) \in \mathcal{N}^{-}$, and

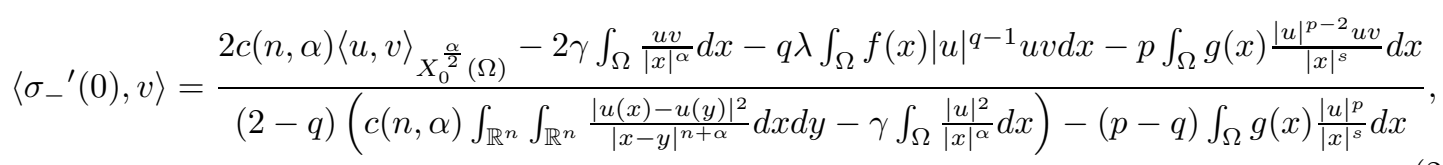

for all $v \in X_{0}^{\frac{\alpha}{2}}(\Omega)$.

Proof. Following the proof of Lemma 3.6, we get that there exist $\epsilon>0$ and a differentiable function $\sigma_{-}: B(0, \epsilon) \longrightarrow \mathbb{R}$ such that $\sigma_{-}(0)=1$ and $\sigma_{-}(v)(u-v) \in \mathcal{N}$, for all $v \in B(0, \epsilon)$. Since $u \in \mathcal{N}^{-}$, we have

$$
\left\langle\phi^{\prime}(u), u\right\rangle=(2-q)\|u\|^{2}-(p-q) \int_{\Omega} g(x) \frac{|u|^{p}}{|x|^{s}} d x<0 .
$$

It then follows from the continuity of $\phi^{\prime}$ and $\sigma_{-}$that

$$
\left\langle\phi^{\prime}\left(\sigma_{-}(v)(u-v)\right), \sigma_{-}(v)(u-v)\right\rangle=(2-q)\left\|\sigma_{-}(v)(u-v)\right\|^{2}-(p-q) \int_{\Omega} g(x) \frac{\left|\sigma_{-}(v)(u-v)\right|^{p}}{|x|^{s}} d x .
$$

Therefore, for $\epsilon>0$ small enough, we get that $\sigma_{-}(v)(u-v) \in \mathcal{N}^{-}$.

Proposition 3.8. Let $\Lambda=\Lambda(p):=\min \left\{\Lambda_{1}, \Lambda_{2}, \Lambda_{3}\right\}$. Then, for any $\lambda \in(0, \Lambda)$, the following hold.

1. There exists a minimizing sequence $\left(u_{k}\right)_{k \in \mathbb{N}} \subset \mathcal{N}$ for $I_{\lambda, p}(u)$ such that

- $I_{\lambda, p}\left(u_{k}\right)=\mathcal{M}+o(1)$.

- $I_{\lambda, p}^{\prime}\left(u_{k}\right)=o(1) \quad$ in $\left(X_{0}^{\frac{\alpha}{2}}(\Omega)\right)^{\prime}$.

2. There exists a minimizing sequence $\left(u_{k}\right)_{k \in \mathbb{N}} \subset \mathcal{N}^{-}$for $I_{\lambda, p}(u)$ such that

- $I_{\lambda, p}\left(u_{k}\right)=\mathcal{M}^{-}+o(1)$.

- $I_{\lambda, p}^{\prime}\left(u_{k}\right)=o(1) \quad$ in $\left(X_{0}^{\frac{\alpha}{2}}(\Omega)\right)^{\prime}$.

Proof. It follows from Lemma 3.5 that $I_{\lambda, p}(u)$ is coercive and bounded below. Then,

1. The Ekeland variational principle implies that there exists a minimizing sequence $\left(u_{k}\right)_{k \in \mathbb{N}}$ such that

$$
I_{\lambda, p}\left(u_{k}\right)<\inf _{\mathcal{N}} I_{\lambda, p}(u)+\frac{1}{k}=\mathcal{M}+\frac{1}{k} \text { and } I_{\lambda, p}\left(u_{k}\right)<I_{\lambda, p}(u)+\frac{1}{k}\left\|u-u_{k}\right\| \quad \text { for all } u \in \mathcal{N} .
$$

For $k$ large enough, we use Lemma 3.5, (12) and (25) to get

$$
\begin{aligned}
\left(\frac{1}{p}-\frac{1}{q}\right) \lambda \int_{\Omega} f(x)\left|u_{k}\right|^{q} d x & \leq I_{\lambda, p}\left(u_{k}\right)=\left(\frac{1}{2}-\frac{1}{p}\right)\left\|u_{k}\right\|^{2}+\left(\frac{1}{p}-\frac{1}{q}\right) \lambda \int_{\Omega} f(x)\left|u_{k}\right|^{q} d x \\
& <\mathcal{M}+\frac{1}{n} \\
& <\mathcal{M} \\
& <\frac{\mathcal{M}}{2} \\
& <0
\end{aligned}
$$


Therefore,

$$
-\frac{\mathcal{M}}{2} \frac{p q}{p-q}<\lambda \int_{\Omega} f(x)\left|u_{k}\right|^{q} d x \leq A S_{p}^{-\frac{q}{2}}\left\|u_{k}\right\|^{q},
$$

which yields $u_{k} \neq 0$, for all $k \in \mathbb{N}$. On the other hand, from (26) and Hölder's inequality, we deduce that

$$
\left\|u_{k}\right\|^{2}<\frac{2 \lambda(p-q)}{q(p-2)} A S_{p}^{-\frac{q}{2}}\left\|w_{k}\right\|^{q}
$$

Hence,

$$
\left(-\frac{\mathcal{M}}{2} \frac{p q}{p-q} A^{-1} S_{p}^{\frac{q}{2}}\right)^{\frac{1}{q}}<\left\|u_{k}\right\|<\left(\frac{2 \lambda(p-q)}{q(p-2)} A S_{p}^{-\frac{q}{2}}\right)^{\frac{1}{2-q}} .
$$

In order to finalize the proof, it is sufficient to show that

$$
\left\|I_{\lambda, p}^{\prime}\left(u_{k}\right)\right\|_{\left(X_{0}^{\frac{\alpha}{2}}(\Omega)\right)^{\prime}} \rightarrow 0 \quad \text { as } k \rightarrow \infty .
$$

Indeed, it follows from Lemma 3.6 that there exists a differentiable function $\sigma_{k}: B_{k}\left(0, \epsilon_{k}\right) \longrightarrow \mathbb{R}_{+}$, for some $\epsilon_{k}$, such that

$$
\sigma_{k}\left(u_{k}-u\right) \in \mathcal{N} \quad \text { for all } k \in \mathbb{N},
$$

where $B_{k}\left(0, \epsilon_{k}\right):=\left\{u \in X_{0}^{\frac{\alpha}{2}}(\Omega):\|u\|<\epsilon_{k}\right\}$. Choose $0<\rho<\epsilon_{k}$, and for any $u \in X_{0}^{\frac{\alpha}{2}}(\Omega) \backslash\{0\}$, define $u_{\rho}:=\frac{\rho u}{\|u\|}$ and $\eta_{\rho}:=\sigma_{k}\left(\rho_{k}\right)\left(u_{k}-u\right)$. Using the fact that $\eta_{\rho} \in \mathcal{N}$, and also $(25)_{2}$, we get that $I_{\lambda, p}\left(u_{k}\right)<I_{\lambda, p}\left(\eta_{\rho}\right)+\frac{1}{k}\left\|\eta_{\rho}-u_{k}\right\|$, which means

$$
I_{\lambda, p}\left(\eta_{\rho}\right)-I_{\lambda, p}\left(u_{k}\right)>-\frac{1}{k}\left\|\eta_{\rho}-u_{k}\right\|
$$

Now we apply the mean value theorem to the left hand-side of the last inequality to deduce

$$
I_{\lambda, p}\left(\eta_{\rho}\right)-I_{\lambda, p}\left(u_{k}\right)=\left\langle I_{\lambda, p}^{\prime}\left(u_{k}\right), \eta_{\rho}-u_{k}\right\rangle+o\left(\left\|\eta_{\rho}-u_{k}\right\|\right)
$$

Thus,

$$
\left\langle I_{\lambda, p}^{\prime}\left(u_{k}\right), \eta_{\rho}-u_{k}\right\rangle+o\left(\left\|\eta_{k}-u_{k}\right\|\right) \geq-\frac{1}{k}\left\|\eta_{\rho}-u_{k}\right\| .
$$

Regarding the first term in (30), we have that

$$
\begin{aligned}
\left\langle I_{\lambda, p}^{\prime}\left(u_{k}\right), \eta_{\rho}-u_{k}\right\rangle & =\left\langle I_{\lambda, p}^{\prime}\left(u_{k}\right), \sigma_{k}\left(u_{\rho}\right)\left(u_{k}-u_{\rho}\right)-u_{k}\right\rangle \\
& =\left\langle I_{\lambda, p}^{\prime}\left(u_{k}\right), \sigma_{k}\left(u_{\rho}\right)\left(u_{k}-u_{\rho}\right)+\left(u_{\rho}-u_{k}\right)-u_{\rho}\right\rangle \\
& =\left\langle I_{\lambda, p}^{\prime}\left(u_{k}\right),-u_{\rho}\right\rangle+\left\langle I_{\lambda, p}^{\prime}\left(u_{k}\right),\left(\sigma_{k}\left(u_{\rho}\right)-1\right)\left(u_{k}-u_{\rho}\right)\right\rangle .
\end{aligned}
$$

Therefore,

$$
\left\langle I_{\lambda, p}^{\prime}\left(u_{k}\right),-u_{\rho}\right\rangle+\left\langle I_{\lambda, p}^{\prime}\left(u_{k}\right),\left(\sigma_{k}\left(u_{\rho}\right)-1\right)\left(u_{k}-u_{\rho}\right)\right\rangle \geq-\frac{1}{k}\left\|\eta_{\rho}-u_{k}\right\|
$$

By the definition of $u_{\rho}$ and $\eta_{\rho}$, we obtain

$$
-\rho\left\langle I_{\lambda, p}^{\prime}\left(u_{k}\right), \frac{u}{\|u\|}\right\rangle+\left(\sigma_{k}\left(u_{\rho}\right)-1\right)\left\langle I_{\lambda, p}^{\prime}\left(u_{k}\right)-I_{\lambda, p}^{\prime}\left(\eta_{\rho}\right),\left(u_{k}-u_{\rho}\right)\right\rangle \geq-\frac{1}{k}\left\|\eta_{\rho}-u_{k}\right\|+o\left(\left\|\eta_{\rho}-u_{k}\right\|\right) .
$$


The last inequality implies that

$$
\left\langle I_{\lambda, p}^{\prime}\left(u_{k}\right), \frac{u}{\|u\|}\right\rangle \leq \frac{1}{\rho k}\left\|\eta_{\rho}-u_{k}\right\|+\frac{o\left(\left\|\eta_{\rho}-u_{k}\right\|\right)}{\rho}+\frac{\left(\sigma_{k}\left(u_{\rho}\right)-1\right)}{\rho}\left\langle I_{\lambda, p}^{\prime}\left(u_{k}\right)-I_{\lambda, p}^{\prime}\left(\eta_{\rho}\right),\left(u_{k}-u_{\rho}\right)\right\rangle .
$$

Note that from Lemma 3.6, it follows

$$
\lim _{\rho \rightarrow 0} \frac{\left|\left(\sigma_{k}\left(u_{\rho}\right)-1\right)\right|}{\rho}=\frac{\left|\left\langle\sigma^{\prime}(0), u_{\rho}\right\rangle\right|}{\rho} \leq\left\|\sigma^{\prime}(0)\right\|
$$

and also simple computations yield

$$
\begin{aligned}
\left\|\eta_{\rho}-u_{k}\right\| & =\left\|\sigma_{k}\left(u_{\rho}\right)\left(u_{k}-u_{\rho}\right) u_{k}\right\| \\
& =\left\|\left(\sigma_{k}\left(u_{\rho}\right)-1\right) u_{k}-u_{\rho} \sigma_{k}\left(u_{\rho}\right)\right\| \\
& \leq\left\|\left(\sigma_{k}\left(u_{\rho}\right)-1\right) u_{k}\right\|+\left\|u_{\rho} \sigma_{k}\left(u_{\rho}\right)\right\| \\
& =\left|\sigma_{k}\left(u_{\rho}\right)-1\right|\left\|u_{k}\right\|+\left|\sigma_{k}\left(u_{\rho}\right)\right| \rho .
\end{aligned}
$$

Using the last two identities, and (32), we then get that

$$
\left\langle I_{\lambda, p}^{\prime}\left(u_{k}\right), \frac{u}{\|u\|}\right\rangle \leq \frac{1}{k}\left|\sigma_{k}\left(u_{\rho}\right)\right|+\frac{1}{k} \frac{\left|\sigma_{k}\left(u_{\rho}\right)-1\right|}{\rho}\left\|u_{k}\right\|+\frac{\left(\sigma_{k}\left(u_{\rho}\right)-1\right)}{\rho}\left\langle I_{\lambda, p}^{\prime}\left(u_{k}\right)-I_{\lambda, p}^{\prime}\left(\eta_{\rho}\right),\left(u_{k}-u_{\rho}\right)\right\rangle .
$$

Taking $\sigma \rightarrow 0$ in the last inequality for a fixed $k$, and using (27), we obtain that there exists a constant $C>0$ (independent of $\rho$ ) such that

$$
\left\langle I_{\lambda, p}^{\prime}\left(u_{k}\right), \frac{u}{\|u\|}\right\rangle \leq \frac{C}{k}\left(1+\left\|\sigma^{\prime}(0)\right\|\right) \quad \text { as } \sigma \rightarrow 0 .
$$

In order to complete the proof of (28), we only need to show that $\left\|\sigma^{\prime}(0)\right\|$ is uniformly bounded in $k$. It follows from (23) and Hölder's inequality that there exists a constant $c>0$ such that

$$
\left\langle\sigma^{\prime}(0), v\right\rangle \leq \frac{c}{\left|(2-q)\left\|u_{k}\right\|^{2}-(p-q) \int_{\Omega} g(x) \frac{\left|u_{k}\right|^{p}}{|x|^{s}} d x\right|} .
$$

It remains to prove that there exists a constant $\bar{c}>0$ such that

$$
\left|(2-q)\left\|u_{k}\right\|^{2}-(p-q) \int_{\Omega} g(x) \frac{\left|u_{k}\right|^{p}}{|x|^{s}} d x\right|>\bar{c} \quad \text { for } n \text { large enough. }
$$

We deduce by contradiction. Suppose that there exists a sub-sequence $\left(u_{k}\right)_{k \in \mathbb{N}}$ such that

$$
(2-q)\left\|u_{k}\right\|^{2}-(p-q) \int_{\Omega} g(x) \frac{\left|u_{k}\right|^{p}}{|x|^{s}} d x=o(1) \quad \text { as } k \rightarrow \infty .
$$

Then, (27) and (34) yield

$$
\begin{aligned}
(p-q) \int_{\Omega} g(x) \frac{\left|u_{k}\right|^{p}}{|x|^{s}} d x & =(2-q)\left\|u_{k}\right\|^{2}+o(1) \\
& \geq(2-q)\left(-\frac{\mathcal{M}}{2} \frac{p q}{p-q} A^{-1} S_{p}^{\frac{q}{2}}\right)^{\frac{1}{q}}+o(1) \quad \text { as } k \rightarrow \infty
\end{aligned}
$$

which implies that there exists a constant $C_{1}>0$ such that 


$$
\int_{\Omega} g(x) \frac{\left|u_{k}\right|^{p}}{|x|^{s}} d x \geq C_{1}>0 .
$$

In addition, by (34) and the fact that $\left(u_{k}\right)_{k \in \mathbb{N}} \in \mathcal{N}$, we have

$$
\begin{aligned}
\lambda \int_{\Omega} f(x)\left|u_{k}\right|^{q} d x & =\left\|u_{k}\right\|^{2}-\int_{\Omega} g(x) \frac{\left|u_{k}\right|^{p}}{|x|^{s}} d x \\
& =\left\|u_{k}\right\|^{2}-\frac{2-q}{p-q}\left\|u_{k}\right\|^{2}+o(1) \\
& =\frac{p-2}{p-q}\left\|u_{k}\right\|^{2}+o(1) \quad \text { as } k \rightarrow \infty .
\end{aligned}
$$

Hence,

$$
\begin{aligned}
\left\|u_{k}\right\| & =\left(\lambda\left(\frac{p-q}{p-2}\right) \int_{\Omega} f(x)\left|u_{k}\right|^{q} d x\right)^{\frac{1}{2}}+o(1) \\
& \leq\left(\lambda\left(\frac{p-q}{p-2}\right) A S_{p}^{-\frac{q}{2}}\right)^{\frac{1}{2-q}}+o(1) \quad \text { as } k \rightarrow \infty .
\end{aligned}
$$

Following the last part of the proof of Lemma 3.1, we get that $J_{\lambda, p}\left(u_{k}\right)=o(1)$ as $k \rightarrow \infty$. On the other hand, we use (35) and the fact that $\lambda \in(0, \Lambda)$ to get

$$
\begin{aligned}
J_{\lambda, p}\left(u_{k}\right) & \geq C(p, q)\left(\frac{\left\|u_{k}\right\|^{2(p-1)}}{\int_{\Omega} g(x) \frac{\left|u_{k}\right|^{p}}{|x|^{s}} d x}\right)^{\frac{1}{p-2}}-\lambda A\left(\int_{\Omega} \frac{\left|u_{k}\right|^{p}}{|x|^{s}} d x\right)^{\frac{q}{p}} \\
& \geq\left(\int_{\Omega} \frac{\left|u_{k}\right|^{p}}{|x|^{s}} d x\right)^{\frac{q}{p}}\left[C(p, q) S_{p}^{\frac{p-1}{p-2}+\frac{q-1}{2-q}}\left(\frac{\lambda A(p-q)}{p-2}\right)^{\frac{1-q}{2-q}}\|g\|_{\infty}^{\frac{1}{2-p}}-\lambda A\right] . \\
& >0,
\end{aligned}
$$

which contradicts $J_{\lambda, p}\left(u_{k}\right)=o(1)$ as $k \rightarrow \infty$. Therefore, (33) holds, and there exists a constant $b>0$ such that

$$
\left\langle I_{\lambda, p}^{\prime}\left(u_{k}\right), \frac{u}{\|u\|}\right\rangle \leq \frac{b}{k} .
$$

This implies (28), and completes the proof.

2. The proof goes exactly as the first part using Lemma 3.7 .

\section{Proof of Theorem 1.1}

In this section, we use the results in section 3 to prove the existence of a positive solution on $\mathcal{N}^{+}$, as well as on $\mathcal{N}^{+}$. This coupled with the fact that $\mathcal{N}^{-} \cap \mathcal{N}^{+}=\emptyset$ yield Theorem 1.1]

Theorem 4.1. Let $\Lambda=\Lambda(p):=\min \left\{\Lambda_{1}, \Lambda_{2}, \Lambda_{3}\right\}$. Then, for any $\lambda \in(0, \Lambda)$, there exists a minimizer $u_{+} \in \mathcal{N}^{+}$for the functional $I_{\lambda, p}$ which verifies

1. $I_{\lambda, p}\left(u_{+}\right)=\mathcal{M}=\mathcal{M}^{+}$.

2. $u_{+}$is positive solution of (1). 
3. $I_{\lambda, p}\left(u_{+}\right) \rightarrow 0 \quad$ as $\lambda \rightarrow 0$.

Proof. Let $\left(u_{k}\right)_{k \in \mathbb{N}} \subset \mathcal{N}$ be a minimizing sequence for $I_{\lambda, p}$ such that $I_{\lambda, p}(u)=\mathcal{M}+o(1)$ and $I_{\lambda, p}^{\prime}(u)=o(1)$ in $\left(X_{0}^{\frac{\alpha}{2}}(\Omega)\right)^{\prime}$, given in the first part of Proposition 3.8, It then follows from Lemma 3.5 and the fractional Sobolev embedding that there exists a sub-sequence $\left(u_{k}\right)_{k \in \mathbb{N}}$ - still denote by $u_{k}$ - and $u_{+} \in X_{0}^{\frac{\alpha}{2}}(\Omega)$ such that

$$
\begin{aligned}
& u_{k} \rightarrow u_{+} \text {weakly in } X_{0}^{\frac{\alpha}{2}}(\Omega) \\
& u_{k} \rightarrow u_{+} \text {strongly in } L^{r}(\Omega) \quad \text { for every } 1 \leq r<2_{\alpha}^{*} .
\end{aligned}
$$

We first show that $\int_{\Omega} f(x)\left|u_{+}\right|^{q} d x \neq 0$. Indeed, suppose $\int_{\Omega} f(x)\left|u_{+}\right|{ }^{q} d x=0$. Then, by (37) 2 , and the fact that $1<q<2<2_{\alpha}^{*}$, we obtain

$$
\int_{\Omega} f(x)\left|u_{k}\right|^{q} d x \rightarrow \int_{\Omega} f(x)\left|u_{+}\right|^{q} d x=0 \quad \text { as } k \rightarrow \infty,
$$

which means

$$
\int_{\Omega} f(x)\left|u_{k}\right|^{q} d x=o(1) \quad \text { as } k \rightarrow \infty
$$

Thus,

$$
\begin{aligned}
\left\|u_{k}\right\|^{2} & =\lambda \int_{\Omega} f(x)\left|u_{k}\right|^{q} d x+\int_{\Omega} g(x) \frac{\left|u_{k}\right|^{p}}{|x|^{s}} d x \\
& =\int_{\Omega} g(x) \frac{\left|u_{k}\right|^{p}}{|x|^{s}} d x+o(1) \quad \text { as } k \rightarrow \infty
\end{aligned}
$$

and

$$
\begin{aligned}
I_{\lambda, p}\left(u_{k}\right) & =\frac{1}{2}\left\|u_{k}\right\|^{2}-\frac{\lambda}{q} \int_{\Omega} f(x)\left|u_{k}\right|^{q} d x-\frac{1}{p} \int_{\Omega} g(x) \frac{\left|u_{k}\right|^{p}}{|x|^{s}} d x \\
& =\left(\frac{1}{2}-\frac{1}{p}\right) \int_{\Omega} g(x) \frac{\left|u_{k}\right|^{p}}{|x|^{s}} d x+o(1) \quad \text { as } k \rightarrow \infty .
\end{aligned}
$$

On the other hand,

$$
I_{\lambda, p}(u)=\mathcal{M}+o(1)<0 \quad \text { as } k \rightarrow \infty .
$$

This leads us to the following contradiction:

$$
0 \leq\left(\frac{p-2}{2 p}\right)\left\|u_{k}\right\|^{2}+o(1)=I_{\lambda, p}\left(u_{k}\right)=\mathcal{M}=o(1)<0 .
$$

Hence,

$$
\int_{\Omega} f(x)\left|u_{+}\right|^{q} d x \neq 0
$$

We now prove that $u_{k} \rightarrow u_{+}$strongly in $X_{0}^{\frac{\alpha}{2}}(\Omega)$. 


$$
\begin{aligned}
\mathcal{M}=\inf _{u \in X_{0}^{\frac{\alpha}{2}}(\Omega) \backslash\{0\}} I_{\lambda, p}(u) & \leq I_{\lambda, p}\left(u_{+}\right)=\frac{1}{2}\left\|u_{+}\right\|^{2}-\frac{\lambda}{q} \int_{\Omega} f(x)\left|u_{+}\right|^{q} d x-\frac{1}{p} \int_{\Omega} g(x) \frac{\left|u_{+}\right|^{p}}{|x|^{s}} d x \\
& =\left(\frac{1}{2}-\frac{1}{p}\right) \int_{\Omega} g(x) \frac{\left|u_{+}\right|^{p}}{|x|^{s}} d x+\left(\frac{1}{p}-\frac{1}{q}\right) \lambda \int_{\Omega} f(x)\left|u_{+}\right|^{q} d x \\
& \leq\left(\frac{1}{2}-\frac{1}{p}\right) \int_{\Omega} g(x) \frac{\left|u_{k}\right|^{p}}{|x|^{s}} d x+\left(\frac{1}{p}-\frac{1}{q}\right) \lambda \int_{\Omega} f(x)\left|u_{k}\right|^{q} d x \\
& =\mathcal{M} .
\end{aligned}
$$

This yields $I_{\lambda, p}\left(u_{+}\right)=\mathcal{M}$, and $u_{k} \rightarrow u_{+}$strongly in $X_{0}^{\frac{\alpha}{2}}(\Omega)$.

The next step is to prove that $u_{+} \in \mathcal{N}^{+}$. Assume that $u_{+} \in \mathcal{N}^{-}$. It then follows from Lemma 3.4 that there exist $t^{-}$and $t^{+}$such that $t^{-} u_{+} \in \mathcal{N}^{-}, t^{+} u_{+} \in N^{+}$and $t^{+}<t^{-}=1$. Following the proof of Lemma 3.4, we have that $\frac{d}{d t} I_{\lambda, p}\left(t^{+} u\right)=0$ and $\frac{d^{2}}{d t^{2}} I_{\lambda, p}\left(t^{+} u\right)>0$. Thus, there exists a $\tilde{t}$ such that $t^{+}<\tilde{t}<t^{-}=1$ and $I_{\lambda, p}\left(t^{+} u_{+}\right)<I_{\lambda, p}\left(\tilde{t} u_{+}\right)$. We again use Lemma 3.4 to get

$$
I_{\lambda, p}\left(t^{+} u_{+}\right)<I_{\lambda, p}\left(\tilde{t} u_{+}\right) \leq I_{\lambda, p}\left(t^{-} u_{+}\right)=I_{\lambda, p}\left(u_{+}\right),
$$

which is in contradiction with $I_{\lambda, p}\left(u_{+}\right)=\mathcal{M}$. Therefore, $u_{+} \in \mathcal{N}^{+}$, and $I_{\lambda, p}\left(u_{+}\right)=\mathcal{M}=\mathcal{M}^{+}$. Since $I_{\lambda, p}\left(u_{+}\right)=I_{\lambda, p}\left(\left|u_{+}\right|\right)$, and $\left|u_{+}\right| \in \mathcal{N}^{+}$is a solution for (1), without loss of generality, we may assume that $u_{+}$is a non-negative solution of (11), and strong maximum principle 23, Proposition 2.2.8] implies that $u_{+}>0$ in $\Omega$.

To complete the proof of Theorem 4.1, we need to show that $I_{\lambda, p}\left(u_{+}\right) \rightarrow 0$ as $\lambda \rightarrow 0$. From Lemma 3.5. it follows

$$
-\lambda\left(\frac{p-q}{p q}\right)\left(\frac{2-q}{2}\right)\left(A S_{p}^{-\frac{q}{2}}\right)^{\frac{2}{2-q}} \leq I_{\lambda, p}\left(u_{+}\right)<\mathcal{M}<0 .
$$

Thus, $I_{\lambda, p}\left(u_{+}\right) \rightarrow 0$ as $\lambda \rightarrow 0$.

Theorem 4.2. Let $\Lambda=\Lambda(p):=\min \left\{\Lambda_{1}, \Lambda_{2}, \Lambda_{3}\right\}$. Then, for any $\lambda \in(0, \Lambda)$, the functional $I_{\lambda, p}$ has a minimizer $u_{-} \in \mathcal{N}^{-}$which verifies

1. $I_{\lambda, p}\left(u_{-}\right)=\mathcal{M}^{-}$.

2. $u_{-}$is a positive solution of (11).

Proof. Let $\left(u_{k}\right)_{k \in \mathbb{N}} \subset \mathcal{N}^{-}$be a minimizing sequence for $I_{\lambda, p}$ such that $I_{\lambda, p}(u)=\mathcal{M}^{-}+o(1)$ and $I_{\lambda, p}^{\prime}(u)=o(1)$ in $\left(X_{0}^{\frac{\alpha}{2}}(\Omega)\right)^{\prime}$, given in the second part of Proposition 3.8. It then follows from Lemma 3.5 and the fractional Sobolev embedding that there exists a sub-sequence $\left(u_{k}\right)_{k \in \mathbb{N}}$ - still denote by $u_{k}$ - and $u_{-} \in X_{0}^{\frac{\alpha}{2}}(\Omega)$ such that

$$
\begin{aligned}
& u_{k} \rightarrow u_{-} \text {weakly in } X_{0}^{\frac{\alpha}{2}}(\Omega) \\
& u_{k} \rightarrow u_{-} \text {strongly in } L^{r}(\Omega) \quad \text { for every } 1 \leq r<2_{\alpha}^{*} .
\end{aligned}
$$

We prove that $u_{k} \rightarrow u_{-}$in $X_{0}^{\frac{\alpha}{2}}(\Omega)$. Indeed, if not, then we have $\left\|u_{-}\right\|<\liminf _{k \rightarrow \infty}\left\|u_{k}\right\|$. Therefore,

$$
\begin{aligned}
\left\langle I_{\lambda, p}^{\prime}\left(u_{-}\right), u_{-}\right\rangle & =\left\|u_{-}\right\|^{2}-\lambda \int_{\Omega} f(x)\left|u_{-}\right|^{q} d x-\int_{\Omega} g(x) \frac{\left|u_{-}\right|^{p}}{|x|^{s}} d x \\
& <\liminf _{k \rightarrow \infty}\left(\left\|u_{k}\right\|^{2}-\lambda \int_{\Omega} f(x)\left|u_{k}\right|^{q} d x-\int_{\Omega} g(x) \frac{\left|u_{k}\right|^{p}}{|x|^{s}} d x\right) \\
& =0,
\end{aligned}
$$


which contradicts $u_{-} \in \mathcal{N}^{-}$. This implies that $u_{k} \rightarrow u_{-}$in $X_{0}^{\frac{\alpha}{2}}(\Omega)$, and therefore $I_{\lambda, p}\left(u_{-}\right)=\mathcal{M}^{-}$. Since $I_{\lambda, p}\left(u_{-}\right)=I_{\lambda, p}\left(\left|u_{-}\right|\right)$, and $\left|u_{-}\right| \in \mathcal{N}^{-}$is a solution for (1), without loss of generality, we may assume that $u_{-}$is a non-negative solution of (1), and the maximum principle [23, Proposition 2.2.8] implies that $u_{-}>0$ in $\Omega$.

Proof of Theorem 1.1. It follows from Theorems 4.1 and 4.2 that there exist two positive solutions $u_{+}$and $u_{-}$such that $u_{+} \in \mathcal{N}^{+}$and $u_{-} \in \mathcal{N}^{-}$. In addition, by Lemma 3.1. $\mathcal{N}^{+} \cap \mathcal{N}^{-}=\emptyset$. Thus, $u_{+}$ and $u_{-}$are two distinct positive solutions for (1).

\section{Proof of Theorem 1.2}

Throughout this section, we shall assume that

$$
p=2_{\alpha}^{*}(s) \quad \text { and } \quad g(x) \equiv 1 .
$$

We also use the following notations for simplicity:

$$
\Lambda^{*}:=\Lambda\left(2_{\alpha}^{*}(s)\right) \quad \text { and } \quad I_{\lambda}(u):=I_{\lambda, 2_{\alpha}^{*}(s)}(u) .
$$

We point out that all results (i.e., Lemmas, Propositions and Theorems) stated in the previous sections hold under condition (39).

The first step to prove Theorem 1.2 is to study the existence and asymptotic behavior of the weak solutions to the following borderline problem associated with the fractional Hardy-Schrödinger operator $(-\Delta)^{\frac{\alpha}{2}}-\frac{\gamma}{|x|^{\alpha}}$ on $\mathbb{R}^{n}$ :

$$
\left\{\begin{aligned}
(-\Delta)^{\frac{\alpha}{2}} u-\gamma \frac{u}{|x|^{\alpha}} & =\frac{u^{2_{\alpha}^{*}(s)-1}}{|x|^{s}} & & \text { in } \mathbb{R}^{n} \\
u & >0 & & \text { in } \mathbb{R}^{n},
\end{aligned}\right.
$$

where $0<\alpha<2,0 \leq s<\alpha, 2_{\alpha}^{*}(s)=\frac{2(n-s)}{n-\alpha}, 0 \leq \gamma<\gamma_{H}(\alpha)=2^{\alpha} \frac{\Gamma^{2}\left(\frac{n+\alpha}{4}\right)}{\Gamma^{2}\left(\frac{n-\alpha}{4}\right)}$.

The existence of the weak solutions to (40) was proved in [13]. Recently, the author and et al. in [14] have proved the following results regarding the asymptotic behavior of such solutions which play a crucial role in this section:

Theorem 5.1 (Theorem 1.2 in [14]). Assume $0 \leq s<\alpha<2, n>\alpha$ and $0 \leq \gamma<\gamma_{H}(\alpha)$. Then, any positive solution $u \in H_{0}^{\frac{\alpha}{2}}\left(\mathbb{R}^{n}\right)$ of (40) satisfies $u \in C^{1}\left(\mathbb{R}^{n} \backslash\{0\}\right)$ and

$$
\lim _{x \rightarrow 0}|x|^{\beta-(\gamma)} u(x)=\lambda_{0} \text { and } \lim _{|x| \rightarrow \infty}|x|^{\beta_{+}(\gamma)} u(x)=\lambda_{\infty},
$$

where $\lambda_{0}, \lambda_{\infty}>0$ and $\beta_{-}(\gamma)$ (resp., $\left.\beta_{+}(\gamma)\right)$ is the unique solution in $\left(0, \frac{n-\alpha}{2}\right)$ (resp., in $\left(\frac{n-\alpha}{2}, n-\alpha\right)$ ) of the equation

$$
\Psi_{n, \alpha}(t):=2^{\alpha} \frac{\Gamma\left(\frac{t+\alpha}{2}\right) \Gamma\left(\frac{n-t}{2}\right)}{\Gamma\left(\frac{n-t-\alpha}{2}\right) \Gamma\left(\frac{t}{2}\right)}=\gamma,
$$

with $\beta_{-}(0)=0$, and $\beta_{+}(0)=n-\alpha$.

We refer the readers to Section 2 in [14] for the definition and properties of $\beta_{+}(\gamma)$ and $\beta_{-}(\gamma)$ in detail

Let $u^{*}(x)$ be a positive weak solution of (3). For any $\epsilon>0$, we define $u_{\epsilon}(x)=\epsilon^{\frac{\alpha-n}{2}} u^{*}\left(\frac{x}{\epsilon}\right)$ in $\mathbb{R}^{n}$. It is easy to show that $u_{\epsilon}(x)$ is also a solution of (3). From the assumption on $f$, we know that $f$ 
is a continuous function, and also $f^{+}(x)=\max \{f(x), 0\} \not \equiv 0$. Let $\Sigma:=\{x \in \Omega: f(x)>0\}$ be an open set of positive measure. Now we need to define appropriate cut-off function. Let $\eta \in C_{0}^{\infty}(\Sigma)$ be a positive cut-off function satisfying $0 \leq \eta \leq 1$ in $\Sigma$. In addition, we choose $\rho>0$ small enough such that $B_{2 \rho}^{c} \subset \bar{\Sigma}, \eta \equiv 1$ in $B_{\rho}$, and $\eta \equiv 0$ in $B_{2 \rho}^{c}$. One can check that $\eta u_{\epsilon}(x)$ is in $X_{0}^{\frac{\alpha}{2}}(\Omega)$. For any $\epsilon>0$, we define

$$
U_{\epsilon}(x)=\eta(x) u_{\epsilon}(x) \quad \text { for } x \in \mathbb{R}^{n} .
$$

The following lemma is a direct consequence of the computations in Section 6.1 in [14]:

Lemma 5.2. Assume that $U_{\epsilon}$ defined as (42), and that $u_{1}$ be a positive solution of (3). Then, for every $\epsilon>0$ small enough, we have

(i) $\left\|U_{\epsilon}\right\|^{2} \leq\left\|u_{\epsilon}\right\|^{2}+O\left(\epsilon^{\beta_{+}(\gamma)-\beta_{-}(\gamma)}\right)$.

(ii) $\int_{\Omega} \frac{\left|U_{\epsilon}\right|_{\alpha}^{2^{*}(s)}}{|x|^{s}} d x=\int_{\Omega} \frac{\left|u_{\epsilon}\right|_{\alpha}^{2_{\alpha}^{*}(s)}}{|x|^{s}} d x+o\left(\epsilon^{\beta_{+}(\gamma)-\beta_{-}(\gamma)}\right)$.

We need the following two lemmas in order to prove Theorem 1.2

Lemma 5.3. Assume that $U_{\epsilon}$ defined as (42), and that $u_{1}$ be the local minimum in Theorem 5.4. Then, for every $\epsilon>0$ small enough, we have

$$
\begin{aligned}
\int_{\Omega} \frac{\left|u_{1}+t U_{\epsilon}\right|_{\alpha}^{*}(s)}{|x|^{s}} d x= & \int_{\Omega} \frac{\left|u_{1}\right|_{\alpha}^{2_{\alpha}^{*}(s)}}{|x|^{s}} d x+\int_{\Omega} \frac{\left|t U_{\epsilon}\right|_{\alpha}^{2_{\alpha}^{*}(s)}}{|x|^{s}} d x+2_{\alpha}^{*}(s) t \int_{\Omega} \frac{\left|u_{1}\right|_{\alpha}^{2_{\alpha}^{*}(s)-2}}{|x|^{s}} U_{\epsilon} u_{1} d x \\
& +2_{\alpha}^{*}(s) t^{2_{\alpha}^{*}(s)-1} \int_{\Omega} \frac{\left|U_{\epsilon}\right|_{\alpha}^{2_{\alpha}^{*}(s)-2}}{|x|^{s}} U_{\epsilon} u_{1} d x+o\left(\epsilon^{\frac{\beta_{+}(\gamma)-\beta_{-}(\gamma)}{2}}\right) .
\end{aligned}
$$

Proof. The proof goes exactly as (17) in [7, Theorem 1] with only minor modifications. We omit it here.

\subsection{The existence of a minimizer on $\mathcal{N}^{+}$}

In the following theorem, we prove the existence of a positive solution of (3) on $\mathcal{N}^{+}$.

Theorem 5.4. For any $\lambda \in\left(0, \Lambda^{*}\right)$, there exists a minimizer $u_{1} \in \mathcal{N}^{+}$for the functional $I_{\lambda}$ which verifies

1. $I_{\lambda}\left(u_{1}\right)=\mathcal{M}=\mathcal{M}^{+}$.

2. $u_{1}$ is positive solution of (3).

3. $I_{\lambda}\left(u_{1}\right) \rightarrow 0 \quad$ as $\lambda \rightarrow 0$.

Proof. The proof is a straightforward consequence of Theorem 4.1 with $p=2_{\alpha}^{*}(s)$.

\subsection{The existence of a minimizer on $\mathcal{N}^{-}$}

In obtaining the existence result on $\mathcal{N}^{-}$, it is crucial to have the (P.S) conditions for all level $\sigma<\mathcal{M}+\frac{\alpha-s}{2(n-s)} S_{p}^{\frac{n-s}{\alpha-s}}$, which will be shown in the next two lemmas.

Lemma 5.5. Let $u_{1}$ be the local minimum in Theorem 5.4. Then, for $\epsilon>0$ small enough, we have

$$
\sup _{t \geq 0} I_{\lambda}\left(u_{1}+t U_{\epsilon}\right)<\mathcal{M}+\frac{\alpha-s}{2(n-s)} S_{p}^{\frac{n-s}{\alpha-s}} .
$$


Proof. We first note that

$$
I_{\lambda}\left(u_{1}+t U_{\epsilon}\right)=\frac{1}{2}\left\|u_{1}+t U_{\epsilon}\right\|^{2}-\frac{\lambda}{q} \int_{\Omega} f(x)\left|u_{1}+t U_{\epsilon}\right|^{q} d x-\frac{1}{2_{\alpha}^{*}(s)} \int_{\Omega} \frac{\left|u_{1}+t U_{\epsilon}\right|^{2_{\alpha}^{*}(s)}}{|x|^{s}} d x .
$$

On the other hand, simple computations yield

$$
\left\|u_{1}+t U_{\epsilon}\right\|^{2}=\left\|u_{1}\right\|^{2}+t^{2}\left\|U_{\epsilon}\right\|^{2}+2 t\left\langle u_{1}, U_{\epsilon}\right\rangle_{X_{0}^{\frac{\alpha}{2}}(\Omega)}-2 \gamma \int_{\Omega} \frac{u_{1} U_{\epsilon}}{|x|^{\alpha}} d x
$$

Thus,

$$
\begin{aligned}
I_{\lambda}\left(u_{1}+t U_{\epsilon}\right) & =\frac{1}{2}\left\|u_{1}\right\|^{2}+\frac{t^{2}}{2}\left\|U_{\epsilon}\right\|^{2}+t\left\langle u_{1}, U_{\epsilon}\right\rangle_{X_{0}^{\frac{\alpha}{2}}(\Omega)}-\gamma \int_{\Omega} \frac{u_{1} U_{\epsilon}}{|x|^{\alpha}} d x \\
& -\frac{\lambda}{q} \int_{\Omega} f(x)\left|u_{1}+t U_{\epsilon}\right|^{q} d x-\frac{1}{2_{\alpha}^{*}(s)} \int_{\Omega} \frac{\left|u_{1}+t U_{\epsilon}\right|_{\alpha}^{*}(s)}{|x|^{s}} d x .
\end{aligned}
$$

Now we deal with each terms separately:

Regarding the first term, since $u_{1}$ is a minimizer for $I_{\lambda}$, we have

$$
\frac{1}{2}\left\|u_{1}\right\|^{2}=I_{\lambda}\left(u_{1}\right)+\frac{\lambda}{q} \int_{\Omega} f(x)\left|u_{1}\right|^{q} d x+\frac{1}{2_{\alpha}^{*}(s)} \int_{\Omega} \frac{\left|u_{1}\right|_{\alpha}^{2_{\alpha}^{*}(s)}}{|x|^{s}} d x .
$$

For the third one, we substitute test function $\eta u_{1}$ into $I_{\lambda}^{\prime}(u)=0$ in $X_{0}^{\frac{\alpha}{2}}(\Omega)$ to get

$$
t\left\langle u_{1}, U_{\epsilon}\right\rangle_{X_{0}^{\frac{\alpha}{2}}(\Omega)}-\gamma \int_{\Omega} \frac{u_{1} U_{\epsilon}}{|x|^{\alpha}} d x=t \lambda \int_{\Omega} f(x)\left|u_{1}\right|^{q-1} U_{\epsilon} d x+t \int_{\Omega} \frac{\left|u_{1}\right|^{2_{\alpha}^{*}(s)-1}}{|x|^{s}} U_{\epsilon} d x .
$$

Plugging the last two inequalities and (43) into (44), we obtain

$$
\begin{aligned}
I_{\lambda}\left(u_{1}+t U_{\epsilon}\right) & =I_{\lambda}\left(u_{1}\right)-\frac{\lambda}{q} \int_{\Sigma}\left(f(x)\left|u_{1}+t U_{\epsilon}\right|^{q} d x-f(x)\left|u_{1}\right|^{q} d x-t q f(x)\left|u_{1}\right|^{q-1} U_{\epsilon}\right) d x \\
& +\frac{t^{2}}{2}\left\|U_{\epsilon}\right\|^{2}-\frac{t_{\alpha}^{2_{\alpha}^{*}(s)}}{2_{\alpha}^{*}(s)} \int_{\Omega} \frac{\left|U_{\epsilon}\right|_{\alpha}^{*}(s)}{|x|^{s}} d x-t^{2_{\alpha}^{*}(s)-1} \int_{\Omega} \frac{\left|U_{\epsilon}\right|^{2_{\alpha}^{*}(s)-1}}{|x|^{s}} u_{1} d x+o\left(\epsilon^{\frac{\beta_{+}(\gamma)-\beta_{-}(\gamma)}{2}}\right) .
\end{aligned}
$$

We also have

$$
\begin{aligned}
& \int_{\Sigma}\left(f(x)\left|u_{1}+t U_{\epsilon}\right|^{q} d x-f(x)\left|u_{1}\right|^{q} d x-t q f(x)\left|u_{1}\right|^{q-1} U_{\epsilon}\right) d x \\
& \quad=q \int_{\Sigma} f(x)\left(\int_{0}^{t U_{\epsilon}}\left|u_{1}+\tau\right|^{q-1}-\left|u_{1}\right|^{q-1} d \tau\right) d x \\
& \quad \geq q \int_{\Sigma} f^{+}(x)\left(\int_{0}^{t U_{\epsilon}}\left|u_{1}+\tau\right|^{q-1}-\left|u_{1}\right|^{q-1} d \tau\right) d x \\
& \quad \geq 0
\end{aligned}
$$

In addition, we know that $u_{1}$ is a positive solution of (3). Following the iterative scheme used to prove Proposition 3.3 in [14, one can show that

$$
u_{1}(x) \leq C|x|^{-\beta_{-}(\gamma)} \quad \text { for all } x \in \Omega
$$

Thus, 


$$
\begin{aligned}
\int_{\Omega} \frac{\left|U_{\epsilon}\right|_{\alpha}^{2_{\alpha}^{*}(s)-1}}{|x|^{s}} u_{1} d x & \leq C \int_{\Omega} \frac{\left|U_{\epsilon}\right|^{2_{\alpha}^{*}(s)-1}}{|x|^{s}}|x|^{-\beta_{-}(\gamma)} d x \\
& =C \int_{B_{\delta}} \frac{\left|U_{\epsilon}\right|_{\alpha}^{2_{\alpha}^{*}(s)-1}}{|x|^{s}}|x|^{-\beta_{-}(\gamma)} d x+C \int_{\Omega_{\backslash B_{\delta}}} \frac{\left|U_{\epsilon}\right|_{\alpha}^{2_{\alpha}^{*}(s)-1}}{|x|^{s}}|x|^{-\beta_{-}(\gamma)} d x \\
& =C \epsilon^{n+\frac{\alpha-n}{2} l-s-\beta_{-}(\gamma)} \int_{B_{\epsilon_{-1}^{-1}}} \frac{\left|u^{*}\right|^{2_{\alpha}^{*}(s)-1}}{|x|^{s}}|x|^{-\beta_{-}(\gamma)} d x+o\left(\epsilon^{\frac{\beta_{+}(\gamma)-\beta_{-}(\gamma)}{2}}\right) \\
& =C \epsilon^{n+\frac{\alpha-n}{2} l-s-\beta_{-}(\gamma)} \int_{\mathbb{R}^{n}} \frac{\left|u^{*}\right|_{\alpha}^{*}(s)-1}{|x|^{s}}|x|^{-\beta_{-}(\gamma)} d x+o\left(\epsilon^{\frac{\beta_{+}(\gamma)-\beta_{-}(\gamma)}{2}}\right) \\
& =C \epsilon^{\frac{\beta_{+}(\gamma)-\beta_{-}(\gamma)}{2}} \int_{\mathbb{R}^{n}} \frac{\left|u^{*}\right|_{\alpha}^{2_{\alpha}^{*}(s)-1}}{|x|^{s}}|x|^{-\beta_{-}(\gamma)} d x+o\left(\epsilon^{\frac{\beta_{+}(\gamma)-\beta_{-}(\gamma)}{2}}\right) \\
& =K \epsilon^{\frac{\beta_{+}(\gamma)-\beta_{-}(\gamma)}{2}}+o\left(\epsilon^{\frac{\beta_{+}(\gamma)-\beta_{-}(\gamma)}{2}}\right) \quad \text { for some } K>0, \quad \text { as } \epsilon \rightarrow 0 .
\end{aligned}
$$

Note that one can use the asymptotic (41) in Theorem [5.1 to show that the last integral is finite. Therefore, there exist $c>0$ such that

$$
I_{\lambda}\left(u_{1}+t U_{\epsilon}\right) \leq I_{\lambda}\left(u_{1}\right)+\frac{t^{2}}{2}\left\|u_{\epsilon}\right\|^{2}-\frac{t^{2_{\alpha}^{*}(s)}}{2_{\alpha}^{*}(s)} \int_{\Omega} \frac{\left|u_{\epsilon}\right|_{\alpha}^{*}(s)}{|x|^{s}} d x-c \epsilon^{\frac{\beta_{+}(\gamma)-\beta_{-}(\gamma)}{2}}+o\left(\epsilon^{\frac{\beta_{+}(\gamma)-\beta_{-}(\gamma)}{2}}\right) .
$$

We now define

$$
m(t)=\frac{t^{2}}{2}\left\|u_{\epsilon}\right\|^{2}-\frac{t_{\alpha}^{2_{\alpha}^{*}(s)}}{2_{\alpha}^{*}(s)} \int_{\Omega} \frac{\left|u_{\epsilon}\right|^{2_{\alpha}^{*}(s)}}{|x|^{s}} d x \quad \text { for } t>0 .
$$

By straightforward computations, we get that $m$ attained its maximum at $\tilde{t}=\left(\frac{\left\|u_{\epsilon}\right\|^{2}}{\int_{\Omega} \frac{\left.\left|u_{\epsilon}\right|\right|_{\alpha} ^{*}(s)}{|x|^{s}} d x}\right)^{\frac{1}{2_{\alpha}^{*}(s)-2}}$, $\lim _{t \rightarrow \infty} m(t)=-\infty$, and also

$$
m(\tilde{t})=\left(\frac{1}{2}-\frac{1}{2_{\alpha}^{*}(s)}\right)\left\|u_{\epsilon}\right\|^{\frac{22_{\alpha}^{*}(s)}{2_{\alpha}^{*}(s)-2}}\left(\int_{\Omega} \frac{\left|u_{\epsilon}\right|_{\alpha}^{*}(s)}{|x|^{s}} d x\right)^{-\frac{2}{2_{\alpha}^{*}(s)-2}} .
$$

Thus, for all $t>0$,

$$
m(t) \leq\left(\frac{1}{2}-\frac{1}{2_{\alpha}^{*}(s)}\right)\left\|u_{\epsilon}\right\|^{\frac{22_{\alpha}^{*}(s)}{2_{\alpha}^{*}(s)-2}}\left(\int_{\Omega} \frac{\left|u_{\epsilon}\right|_{\alpha}^{*}(s)}{|x|^{s}} d x\right)^{-\frac{2}{2_{\alpha}^{*}(s)-2}} .
$$

On the other hand, since $u_{\epsilon}$ is an extremal for (8), we have

$$
\left\|u_{\epsilon}\right\|^{2}=S_{p}\left(\int_{\Omega} \frac{\left|u_{\epsilon}\right|^{2}(s)}{|x|^{s}} d x\right)^{\frac{2}{2 *(s)}} .
$$

Hence,

$$
S_{p}^{\frac{2_{\alpha}^{*}(s)}{2_{\alpha}^{*}(s)-2}}=\left\|u_{\epsilon}\right\|^{\frac{22_{\alpha}^{*}(s)}{2_{\alpha}^{*}(s)-2}}\left(\int_{\Omega} \frac{\left|u_{\epsilon}\right|_{\alpha}^{*}(s)}{|x|^{s}} d x\right)^{-\frac{2}{2_{\alpha}^{*}(s)-2}} .
$$

Noting that $\frac{1}{2}-\frac{1}{2_{\alpha}^{*}(s)}=\frac{\alpha-s}{2(n-s)}$ and $\frac{2_{\alpha}^{*}(s)}{2_{\alpha}^{*}(s)-2}=\frac{n-s}{\alpha-s}$, we get

$$
m(t) \leq \frac{\alpha-s}{2(n-s)} S_{p}^{\frac{n-s}{\alpha-s}} \quad \text { for all } t>0 .
$$


Therefore,

$$
\begin{aligned}
I_{\lambda}\left(u_{1}+t U_{\epsilon}\right) & \leq I_{\lambda}\left(u_{1}\right)+\frac{\alpha-s}{2(n-s)} S_{p}^{\frac{n-s}{\alpha-s}}-c \epsilon^{\frac{\beta_{+}(\gamma)-\beta_{-}(\gamma)}{2}}+o\left(\epsilon^{\frac{\beta_{+}(\gamma)-\beta_{-}(\gamma)}{2}}\right) \\
& <\mathcal{M}+\frac{\alpha-s}{2(n-s)} S_{p}^{\frac{n-s}{\alpha-s}} \quad \text { for all } t>0
\end{aligned}
$$

Lemma 5.6. Suppose that a sequence $\left(u_{k}\right)_{k \in \mathbb{N}}$ satisfies the following:

1. $I_{\lambda}\left(u_{k}\right)=\sigma+o(1) \quad$ with $\sigma<\mathcal{M}+\frac{\alpha-s}{2(n-s)} S_{p}^{\frac{n-s}{\alpha-s}}$

2. $I_{\lambda}^{\prime}\left(u_{k}\right)=o(1) \quad$ in $\left(X_{0}^{\frac{\alpha}{2}}(\Omega)\right)^{\prime}$

Then, there exists a sub-sequence of $\left(u_{k}\right)_{k \in \mathbb{N}}$ which is strongly convergence in $X_{0}^{\frac{\alpha}{2}}(\Omega)$.

Proof. It follows from Lemma 3.5 that $\left(u_{k}\right)_{k \in \mathbb{N}}$ is bounded in $X_{0}^{\frac{\alpha}{2}}(\Omega)$. Then, there exists a subsequence - still donote by $u_{k}$ - and $u$ such that

$$
\begin{aligned}
& u_{k} \rightarrow u \text { weakly in } X_{0}^{\frac{\alpha}{2}}(\Omega) \\
& u_{k} \rightarrow u \text { strongly in } L^{r}(\Omega) \quad \text { for every } 1 \leq r<2_{\alpha}^{*} .
\end{aligned}
$$

Consequently from the second assumption, we obtain

$$
\left\langle I_{\lambda}^{\prime}(u), w\right\rangle=0 \quad \forall w \in X_{0}^{\frac{\alpha}{2}}(\Omega)
$$

Then, $u$ is a solution in $X_{0}^{\frac{\alpha}{2}}(\Omega)$ for (3) with $I_{\lambda}(u) \geq \mathcal{M}$.

We first prove that $u \not \equiv 0$. Indeed, suppose $u \equiv 0$. Then, by (46]) 2 , and the fact that $1<q<2<2_{\alpha}^{*}$, we obtain

$$
\int_{\Omega} f(x)\left|u_{k}\right|^{q} d x \rightarrow \int_{\Omega} f(x)|u|^{q} d x=0
$$

which implies

$$
\int_{\Omega} f(x)\left|u_{k}\right|^{q} d x=o(1) \quad \text { as } k \rightarrow \infty .
$$

Thus, the second assumption yields

$$
\begin{aligned}
\left\|u_{k}\right\|^{2} & =\lambda \int_{\Omega} f(x)\left|u_{k}\right|^{q} d x+\int_{\Omega} \frac{\left|u_{k}\right|^{2_{\alpha}^{*}(s)}}{|x|^{s}} d x \\
& =\int_{\Omega} \frac{\left|u_{k}\right|^{2_{\alpha}^{*}(s)}}{|x|^{s}} d x+o(1) \quad \text { as } k \rightarrow \infty
\end{aligned}
$$

and the first assumption then implies that

$$
\begin{aligned}
I_{\lambda, p}\left(u_{k}\right) & =\frac{1}{2}\left\|u_{k}\right\|^{2}-\frac{\lambda}{q} \int_{\Omega} f(x)\left|u_{k}\right|^{q} d x-\frac{1}{2_{\alpha}^{*}(s)} \int_{\Omega} \frac{\left|u_{k}\right|^{2_{\alpha}^{*}(s)}}{|x|^{s}} d x \\
& =\left(\frac{1}{2}-\frac{1}{2_{\alpha}^{*}(s)}\right) \int_{\Omega} \frac{\left|u_{k}\right|^{2_{\alpha}^{*}(s)}}{|x|^{s}} d x+o(1) \\
& =\frac{\alpha-s}{2(n-s)} \int_{\Omega} \frac{\left|u_{k}\right|^{2_{\alpha}^{*}(s)}}{|x|^{s}} d x+o(1) \\
& =\sigma+o(1) \quad \text { as } k \rightarrow \infty .
\end{aligned}
$$


Since $\sigma<\frac{\alpha-s}{2(n-s)} S_{p}^{\frac{n-s}{\alpha-s}}$, we get that

$$
\int_{\Omega} \frac{\left|u_{k}\right|^{2_{\alpha}^{*}(s)}}{|x|^{s}} d x<S_{p}^{\frac{n-s}{\alpha-s}}+o(1) \quad \text { as } k \rightarrow \infty .
$$

On the other hand, it follows from (8) and (47) that

$$
\int_{\Omega} \frac{\left|u_{k}\right|_{\alpha}^{*}(s)}{|x|^{s}} d x \geq S_{p}^{\frac{n-s}{\alpha-s}}+o(1) \quad \text { as } k \rightarrow \infty .
$$

This gives us a contradiction which implies that $u$ can not be identically zero, and thus $u \not \equiv 0$ with $I_{\lambda}(u) \geq \mathcal{M}$.

Let now $v_{k}=u_{k}-u$, for all $k \in \mathbb{N}$. We may verify as Brzis-Lieb lemma in [6] that (see also [15, Lemma 4.2])

$$
\int_{\Omega} \frac{\left|u_{k}\right|_{\alpha}^{2_{\alpha}^{*}(s)}}{|x|^{s}} d x=\int_{\Omega} \frac{|u|_{\alpha}^{2_{\alpha}^{*}(s)}}{|x|^{s}} d x+\int_{\Omega} \frac{\left|v_{k}\right|_{\alpha}^{*}(s)}{|x|^{s}} d x+o(1) \quad \text { as } k \rightarrow \infty .
$$

Hence, by weakly convergence $v_{k} \rightarrow 0$ in $X_{0}^{\frac{\alpha}{2}}(\Omega)$, we can conclude that

$$
\begin{aligned}
\mathcal{M}+\frac{\alpha-s}{2(n-s)} S_{p}^{\frac{n-s}{\alpha-s}} & >I_{\lambda}\left(u_{2}+v_{k}\right) \\
& =I_{\lambda}\left(u_{2}\right)+\frac{1}{2}\left\|v_{k}\right\|^{2}-\frac{1}{2_{\alpha}^{*}(s)} \int_{\Omega} \frac{\left|v_{k}\right|^{2 *}(s)}{|x|^{s}} d x+o(1) \\
& \geq \mathcal{M}+\frac{1}{2}\left\|v_{k}\right\|^{2}-\frac{1}{2_{\alpha}^{*}(s)} \int_{\Omega} \frac{\left|v_{k}\right|_{\alpha}^{*}(s)}{|x|^{s}} d x+o(1) \quad \text { as } k \rightarrow \infty .
\end{aligned}
$$

Then,

$$
\frac{1}{2}\left\|v_{k}\right\|^{2}-\frac{1}{2_{\alpha}^{*}(s)} \int_{\Omega} \frac{\left|v_{k}\right|^{2 *}(s)}{|x|^{s}} d x<\frac{\alpha-s}{2(n-s)} S_{p}^{\frac{n-s}{\alpha-s}}+o(1) \quad \text { as } k \rightarrow \infty .
$$

On the other hand, from the second assumption, we know that $\left(u_{k}\right)_{k \in \mathbb{N}}$ is uniformly bounded and $u$ is solution of (3). So,

$$
\begin{aligned}
o(1) & =I_{\lambda}^{\prime}\left(u_{k}, u_{k}\right) \\
& =\frac{1}{2}\left\|u_{k}\right\|^{2}-\lambda \int_{\Omega} f(x)\left|u_{k}\right|^{q} d x-\int_{\Omega} \frac{\left|u_{k}\right|^{2_{\alpha}^{*}(s)}}{|x|^{s}} d x \\
& =I_{\lambda}^{\prime}(u)+\left\|v_{k}\right\|^{2}-\int_{\Omega} \frac{\left|v_{k}\right|^{2_{\alpha}^{*}(s)}}{|x|^{s}} d x+o(1) \quad \text { as } k \rightarrow \infty .
\end{aligned}
$$

Since $I_{\lambda}^{\prime}(u)=0$, we have

$$
\left\|v_{k}\right\|^{2}-\int_{\Omega} \frac{\left|v_{k}\right|_{\alpha}^{*}(s)}{|x|^{s}} d x=o(1) \quad \text { as } k \rightarrow \infty .
$$

Now we prove that if (48) and (49) hold, then $\left(v_{k}\right)_{k \in \mathbb{N}}$ admits a sub-sequence which converges strongly to zero. Indeed, if not, there exits a constant $c>0$ such that $\left\|v_{k}\right\|_{X_{0}^{\frac{\alpha}{2}}(\Omega)}^{2} \geq c>0$, for all $k \in \mathbb{N}$. Combining (48) and (49) leads us to the following contradiction: 


$$
\begin{aligned}
\frac{\alpha-s}{2(n-s)} S_{p}^{\frac{n-s}{\alpha-s}} & \leq \frac{\alpha-s}{2(n-s)}\left\|v_{k}\right\|^{2}+o(1) \\
& =\frac{1}{2}\left\|v_{k}\right\|_{X_{0}^{\frac{\alpha}{2}}(\Omega)}^{2}-\frac{1}{2_{\alpha}^{*}(s)}\left\|v_{k}\right\|^{2}+o(1) \\
& <\frac{\alpha-s}{2(n-s)} S_{p}^{\frac{n-s}{\alpha-s}}+o(1) \quad \text { as } k \rightarrow \infty .
\end{aligned}
$$

Therefore, up to a sub-sequence, $v_{k} \rightarrow 0$ strongly in $X_{0}^{\frac{\alpha}{2}}(\Omega)$. This implies that $u_{k} \rightarrow u$ strongly in $X_{0}^{\frac{\alpha}{2}}(\Omega)$.

We are now ready to prove the existence results on $\mathcal{N}^{+}$.

Proposition 5.7. For any $\lambda \in\left(0, \Lambda^{*}\right)$, there exists a minimizer $u_{2} \in \mathcal{N}^{-}$for the functional $I_{\lambda}$ which verifies

1. $I_{\lambda}\left(u_{2}\right)=\mathcal{M}^{-}<\mathcal{M}+\frac{\alpha-s}{2(n-s)} S_{p}^{\frac{n-s}{\alpha-s}}$.

2. $u_{2}$ is a nontrivial non-negative solution of (3).

Proof. We first show that

$$
\mathcal{M}^{-}<\mathcal{M}+\frac{\alpha-s}{2(n-s)} S_{p}^{\frac{n-s}{\alpha-s}}
$$

Let

$$
W_{1}:=\left\{u \in X_{0}^{\frac{\alpha}{2}}(\Omega) \backslash\{0\}: t^{-}\left(\frac{u}{\|u\|}\right)>\|u\|\right\} \cup\{0\},
$$

and

$$
W_{2}:=\left\{u \in X_{0}^{\frac{\alpha}{2}}(\Omega) \backslash\{0\}: t^{-}\left(\frac{u}{\|u\|}\right)<\|u\|\right\} .
$$

Thus, $\mathcal{N}^{-}$disconnects $X_{0}^{\frac{\alpha}{2}}(\Omega)$ in two connected components $W_{1}$ and $W_{2}$, and $X_{0}^{\frac{\alpha}{2}}(\Omega) \backslash \mathcal{N}^{-}=W_{1} \cup$ $W_{2}$. By Lemma 3.4, for any $u \in \mathcal{N}^{+}$, there exists a unique $t^{-}\left(\frac{u}{\|u\|}\right)>0$ such that $1<t_{\max }<t^{-}(u)$. Since $t^{-}(u)=\frac{1}{\|u\|} t^{-}\left(\frac{u}{\|u\|}\right)$. Then, $t^{-}\left(\frac{u}{\|u\|}\right)>\|u\|$, and $\mathcal{N}^{+} \subset W_{1}$. In particular, $u_{1} \in W_{1}$.

Next step is to show that there exists $n_{0}>0$ such that $u_{1}+n_{0} U_{\epsilon} \in W_{2}$. To prove this, we first note that there exists $C>0$ such that

$$
0<t^{-}\left(\frac{u_{1}+n_{0} U_{\epsilon}}{\left\|u_{1}+n_{0} U_{\epsilon}\right\|}\right)<C \quad \text { for all } n_{0}>0 .
$$

Indeed, if not, there exists a sub-sequence $\left(n_{k}\right)_{k \in \mathbb{N}}$ such that

$$
n_{k} \rightarrow \infty \quad \text { and } \quad t^{-}\left(\frac{u_{1}+n_{k} U_{\epsilon}}{\left\|u_{1}+n_{k} U_{\epsilon}\right\|}\right) \rightarrow 0 \quad \text { as } k \rightarrow \infty .
$$

For all $k \in \mathbb{N}$, let $v_{k}=\frac{u_{1}+n_{k} U_{\epsilon}}{\left\|u_{1}+n_{k} U_{\epsilon}\right\|}$. So, Lemma 3.4 implies that $t^{-}\left(v_{k}\right) v_{k} \in \mathcal{N}^{-} \subset \mathcal{N}$ for all $k \in \mathcal{N}$. Then, a straightforward computation and the Lebesgue dominated convergence theorem yield 


$$
\begin{aligned}
\int_{\Omega} \frac{\left|v_{k}\right|^{2_{\alpha}^{*}(s)}}{|x|^{s}} d x & =\frac{1}{\left\|u_{1}+n_{k} U_{\epsilon}\right\|^{2_{\alpha}^{*}(s)}} \int_{\Omega} \frac{\left|u_{1}+n_{k} U_{\epsilon}\right|^{2_{\alpha}^{*}(s)}}{|x|^{s}} d x \\
& =\frac{1}{\left\|\frac{u_{1}}{n_{k}}+U_{\epsilon}\right\|^{2 *}(s)} \int_{\Omega} \frac{\left|\frac{u_{1}}{n_{k}}+U_{\epsilon}\right|^{2^{*}(s)_{\alpha}}}{|x|^{s}} d x .
\end{aligned}
$$

Hence,

$$
\int_{\Omega} \frac{\left|v_{k}\right|_{\alpha}^{2_{\alpha}^{*}(s)}}{|x|^{s}} d x \rightarrow \frac{1}{\left\|U_{\epsilon}\right\|^{2 *(s)}} \int_{\Omega} \frac{\left|U_{\epsilon}\right|^{2^{*}(s)_{\alpha}}}{|x|^{s}} d x>0 \quad \text { as } k \rightarrow \infty .
$$

On the other hand, as $k \rightarrow \infty$, we have

$$
\begin{aligned}
& I_{\lambda}\left(t^{-}\left(v_{k}\right) v_{k}\right) \\
& \quad=\frac{1}{2}\left[t^{-}\left(v_{k}\right)\right]^{2}\left\|v_{k}\right\|^{2}-\frac{\lambda}{q}\left[t^{-}\left(v_{k}\right)\right]^{q} \int_{\Omega} f(x)\left|v_{k}\right|^{q} d x-\frac{1}{2_{\alpha}^{*}(s)}\left[t^{-}\left(v_{k}\right)\right]^{2_{\alpha}^{*}(s)} \int_{\Omega} \frac{\left|v_{k}\right|_{\alpha}^{2_{\alpha}^{*}(s)}}{|x|^{s}} d x \rightarrow-\infty .
\end{aligned}
$$

This contradicts the fact that $I_{\lambda}$ is bounded below. Thus, (51) holds.

Now let $n_{0}:=\frac{\left|C^{2}-\left\|u_{1}\right\|^{2}\right|^{\frac{1}{2}}}{\left\|U_{\epsilon}\right\|}+1$. So,

$$
\begin{aligned}
\left\|u_{1}+n_{0} U_{\epsilon}\right\|^{2} & =\left\|u_{1}\right\|^{2}+n_{0}^{2}\left\|U_{\epsilon}\right\|^{2}+2 n_{0}\left(C_{n, \alpha}\left\langle u_{1}, U_{\epsilon}\right\rangle_{X_{0}^{2}}^{\frac{\alpha}{2}(\Omega)}-\gamma \int_{\Omega} \frac{u_{1} U_{\epsilon}}{|x|^{\alpha}} d x\right) \\
& \geq\left\|u_{1}\right\|^{2}+\left|C^{2}-\left\|u_{1}\right\|^{2}\right| \\
& \geq C^{2} \\
& >\left|t^{-}\left(\frac{u_{1}+n_{0} U_{\epsilon}}{\left\|u_{1}+n_{0} U_{\epsilon}\right\|}\right)\right|^{2}
\end{aligned}
$$

which gives

$$
t^{-}\left(\frac{u_{1}+n_{0} U_{\epsilon}}{\left\|u_{1}+n_{0} U_{\epsilon}\right\|}\right)<\left\|u_{1}+n_{0} U_{\epsilon}\right\|
$$

This proves that $u_{1}+n_{0} U_{\epsilon} \in W_{2}$.

Now define

$$
\begin{gathered}
\Gamma:=\left\{\tau \in C\left([0,1], X_{0}^{\frac{\alpha}{2}}(\Omega)\right): \tau(0)=u_{1} \text { and } \tau(1)=u_{1}+n_{0} U_{\epsilon}\right\}, \\
c^{\star}:=\inf _{\tau \in \Gamma} \max _{\xi \in[0,1]} I_{\lambda}(\tau(\xi)) \text { and } \gamma^{\star}(\xi)=u_{1}+\xi n_{0} U_{\epsilon} \quad \text { for } \xi \in[0,1] .
\end{gathered}
$$

We have $\gamma^{\star}(0) \in W_{1}$ and $\gamma^{\star}(1) \in W_{2}$. So, there exists $\xi_{0} \in(0,1)$ such that $\gamma^{\star}\left(\xi_{0}\right) \in \mathcal{N}^{-}$and $c^{\star} \geq \mathcal{M}^{-}$. It also follows from Lemma [5.6 that

$$
\mathcal{M}^{-}<c^{\star}<\mathcal{M}+\frac{\alpha-s}{2(n-s)} S_{p}^{\frac{n-s}{\alpha-s}}
$$

The Ekeland's variational principle yields that there exists a sequence $\left(u_{k}\right)_{k \in \mathbb{N}} \subset \mathbb{N}^{-}$such that

$$
I_{\lambda}\left(u_{k}\right)=\mathcal{M}^{-}+o(1) \quad \text { and } I_{\lambda}^{\prime}\left(u_{k}\right)=o(1) \text { in }\left(X_{0}^{\frac{\alpha}{2}}(\Omega)\right)^{\prime} .
$$


We use Lemma 5.6 and (50) to get that there exist a sub-sequence $\left(u_{k}\right)_{k \in \mathbb{N}}$ and $u_{2}$ such that $u_{k} \rightarrow u_{2}$ strongly in $X_{0}^{\frac{\alpha}{2}}(\Omega)$. So, we have that $u_{2} \in \mathcal{N}^{-}$and $I_{\lambda}\left(u_{k}\right) \rightarrow I_{\lambda}\left(u_{2}\right)=\mathcal{M}^{-}$as $k \rightarrow \infty$.

Since $I_{\lambda}\left(u_{2}\right)=I_{\lambda}\left(\left|u_{2}\right|\right)$, and $\left|u_{2}\right| \in \mathcal{N}^{-}$is a solution for (3), without loss of generality, we may assume that $u_{2}$ is a non-negative solution for (3), and the maximum principle [23. Proposition 2.2.8] implies that $u_{2}>0$ in $\Omega$.

Proof of Theorem 1.2. It follows from Theorem 5.4 and Proposition 5.7 that there exist two positive solutions $u_{1}$ and $u_{2}$ such that $u_{1} \in \mathcal{N}^{+}$and $u_{2} \in \mathcal{N}^{-}$. In addition, we have $\mathcal{N}^{+} \cap \mathcal{N}^{-}=\emptyset$. Thus, $u_{1}$ and $u_{2}$ are two distinct positive solutions for (3).

\section{References}

[1] M. Bhakta and D. Mukherjee, Multiplicity results and sign changing solutions of non-local equations with concave-convex nonlinearities, arXiv preprint arXiv:1603.05554 (2016).

[2] B. Barrios, E. Colorado, A. de Pablo, and U. S'anchez, On some critical problems for the fractional Laplacian operator. J. Diff. Eqns 252 (2012), 6133-6162.

[3] C. Brandle, E. Colorado, A. De Pablo and U. S'anchez, A concave-convex elliptic problem involving the fractional Laplacian. Proc. Roy. Soc. Edinburgh Sect. A, 143 (2013), 39-71.

[4] B. Barrios, M. Medina and I. Peral, Some remarks on the solvability of non-local elliptic problems with the Hardy potential, Communications in Contemporary Mathematics 16.04 (2014): 1350046 .

[5] G.M. Bisci, Giovanni Molica, V. D. Radulescu and R. Servadei, Variational methods for nonlocal fractional problems. Vol. 162. Cambridge University Press, 2016.

[6] H. Brezis and E. Lieb, A Relation Between Point Convergence of Functions and Convergence of Functionals, Proc. Amer. Math. Soc. 88(1983), 486-490. MR 84c:28003

[7] H. Brezis and L. Nirenberg, A minimization problem with critical exponent and non-zero data, Sc. Norm. Super. di Pisa Quaderni (1989), pp. 129140

[8] H. Brezis and L. Nirenberg, Positive solutions of nonlinear elliptic equations involving critical Sobolev exponents, Comm. Pure Appl. Math. 36 (4) (1983) 437-477.

[9] L. Caffarelli and L. Silvestre, An extension problem related to the fractional Laplacian, Comm. in Part. Diff. Equa. 32 (2007), 1245-1260.

[10] A. Cotsiolis and N.K. Tavoularis, Best constants for Sobolev inequalities for higher order fractional derivatives J. Math. Anal. Appl., 295 (2004), pp. 225-236.

[11] E. Di Nezza, G. Palatucci and E. Valdinoci, Hitchhikers guide to the fractional Sobolev spaces, Bull. Sci. Math. 136 (2012), no. 5, 521-573. 687-698, 1988.

[12] R. L. Frank, E. H. Lieb and R. Seiringer, Hardy-Lieb-Thirring inequalities for fractional Schrdinger operators, J. Amer. Math. Soc. 21(4) (2008) 925-950.

[13] N. Ghoussoub and S. Shakerian, Borderline variational problems involving fractional Laplacians and critical singularities, Advanced Nonlinear Studies, 15 (2015) 527-555 
[14] N. Ghoussoub, F. Robert, S. Shakerian and M. Zhao, Mass and Asymptotics Associated to Fractional Hardy-Schrödinger Operators in Critical Regimes, arXiv preprint arXiv:1704.08658 (2017).

[15] N. Ghoussoub and C. Yuan, Multiple solutions for quasi-linear PDEs involving the critical Sobolev and Hardy exponents, Trans. Amer. Math. Soc. 12 (2000), 5703-5743.

[16] I. Herbst, Spectral theory of the operator $\left(p^{2}+m^{2}\right)^{1 / 2}-Z e^{2} / r$, Communications in Mathematical Physics 53.3 (1977): 285-294.

[17] A. Quaas and A. Xia, Multiple positive solutions for nonlinear critical fractional elliptic equations involving sign-changing weight functions, arXiv preprint arXiv:1602.08276 (2016).

[18] L. Wang, Q. Wei, and D. Kang, Multiple positive solutions for p-Laplace elliptic equations involving concaveconvex nonlinearities and a Hardy-type term, Nonlinear Analysis: Theory, Methods \& Applications 74.2 (2011): 626-638.

[19] T. F. Wu, On semilinear elliptic equations involving concaveconvex nonlinearities and signchanging weight function. Journal of Mathematical Analysis and Applications 318.1 (2006): 253-270.

[20] T. F. Wu, On semilinear elliptic equations involving critical Sobolev exponents and signchanging weight function (2008).

[21] R. Servadei and E. Valdinoci, The Brezis-Nirenberg result for the fractional Laplacian, Transactions of the American Mathematical Society 367.1 (2015): 67-102.

[22] Z. Shen and F. Gao, Existence of solutions for a fractional Laplacian equation with critical nonlinearity. Abstract Appl. Anal. 2013;2013, Atc. ID 638425, 9 pp.

[23] L. Silvestre, Regularity of the obstacle problem for a fractional power of the Laplace operator. Comm. Pure Appl. Math., 60(1):67-112, 2007.

[24] G. Tarantello, On nonhomogeneous elliptic equations involving critical Sobolev exponent." Annales de l'IHP Analyse non linéaire. Vol. 9. No. 3. 1992.

[25] J. Zhang, X. Liu and H. Jiao, Multiplicity of positive solutions for a fractional Laplacian equations involving critical nonlinearity, arXiv preprint arXiv:1502.02222 (2015). 